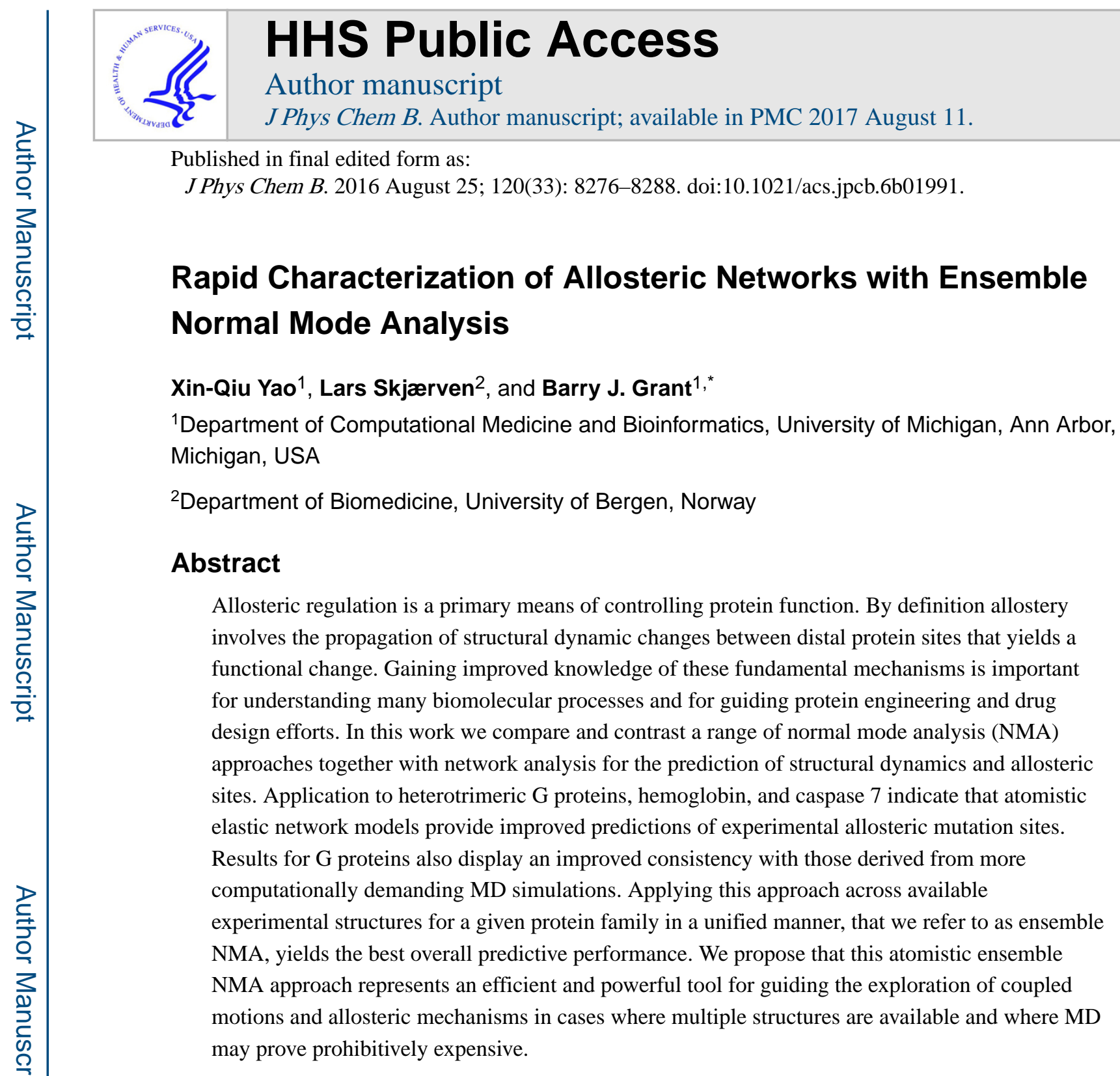

\title{
TOC image
}

"Corresponding Author: B. J. Grant, 100 Washtenaw Ave., 2017 Palmer Commons Bldg., Ann Arbor, MI 48109-2218, Tel: 734-647-3113,bjgrant@umich.edu.

Supporting Information: Figures S1-S12, Tables S1-S4.

Author Contributions

BJG and XQY designed the research. XQY and LS performed calculations. XQY, LS and BJG developed tools, analyzed results and wrote the manuscript. All authors have given approval to the final version of the manuscript. 


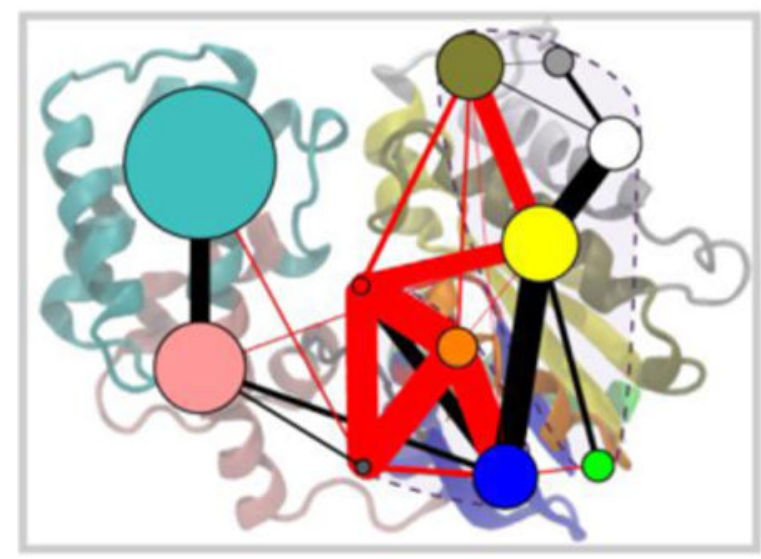

\section{Introduction}

Allostery, the coupling of structural dynamic changes at distal binding interfaces, regulates numerous biomolecular processes. Typically manifested as an allosteric effector modulating the behavior of a spatially distant orthosteric active site, this ubiquitous form of intramolecular communication provides a critical means by which the environment can affect cellular function. Indeed, many events can serve as allosteric effectors including the binding of a second molecule, a change in ionic strength or $\mathrm{pH}$, as well as modifications such as mutation, phosphorylation, ubiquitination and glycosylation. Assessing and predicting the allosteric consequences of such events is challenging due in large part to the difficulty associated with fully characterizing the underlying atomic motions and their couplings. Gaining an improved understanding of these motions and developing the ability to predict allosteric sites has significant potential for facilitating the development of new drugs and regulators of biochemical (mis)function in healthy and disease states ${ }^{1-2}$.

A number of recently developed computational methods for the characterization and prediction of allosteric mechanisms exploit a common network analysis approach. These methods, typically based on molecular dynamics (MD) simulations, aim to dissect dynamic residue couplings, energetic coupling, or coordinated contact rearrangement have been recently applied to elucidate allosteric mechanisms in a number of diverse protein systems $^{3-9}$. Notably, adopting this approach we have recently characterized nucleotide dependent networks of residue correlations in the a subunit of heterotrimeric $G$ proteins $(\mathrm{Ga})$. This work revealed how allosteric signals propagate between distal receptor, nucleotide and domain-domain interfaces to modulate activity ${ }^{8}$. In particular, we have predicted and characterized novel point mutations that affect these allosteric networks leading to enhanced $\mathrm{Ga}$ activation. These mutants have been further validated by experimental mutagenesis to have increased nucleotide exchange rates leading to constitutive activity. This and other examples previously noted indicate that network analysis based on MD simulations can be useful for identifying allosteric sites where mutations or binding of other molecules may affect protein function allosterically. However, a major limitation to the further wide spread adoption of this overall approach is the typical requirement for multiple long time $(\sim 100 \mathrm{~ns})$ simulation replicates. Maintaining prediction 
accuracy whilst alleviating both the significant hardware and physical time demands associated with these simulations would greatly facilitate the productive study of allostery in a boarder range of biomolecular systems.

A more rapid alternative computational approach for deriving information on protein intrinsic dynamics exploits normal mode analysis (NMA). NMA, especially when combined with a coarse-grained elastic network model (ENM) formulation, can in principle generate the dynamic information required for a similar network analysis in seconds - many orders of magnitude faster than MD. The overall consistency between the simplified NMA approach and either MD or experiment has been previously documented in numerous studies ${ }^{10-11}$. Predicted motions derived from low frequency normal modes have been shown to match the conformational transitions revealed by multiple crystallographic structures solved under distinct conditions ${ }^{10}$. In addition, the overall profile of residue-wise atomic fluctuations and covariance estimates, which contain contributions from both slow and fast normal modes, has recently been shown to be highly similar between MD and a number of popular ENM approaches ${ }^{10-11}$. However, it is still unclear whether any ENM model is sufficiently accurate at the higher level of precision typically required for predictive allosteric network analysis. In particular it is currently unclear whether ENMs can capture the essential features, in terms of allosteric residue level predictions, obtained from extensive MD simulations of $\mathrm{Ga}$ and other known allosteric proteins.

In this work, we first test the performance of several popular ENMs on the prediction of fluctuations, covariance, and correlation networks in Ga. We apply the ENM NMA to all the available Ga crystallographic structures. This ensemble based NMA approach is shown to have overall improved performance in relation to any single structure NMA calculation for all ENMs tested. We show that in spite of the overall agreement with MD in terms of fluctuations and covariance, conventional ENMs fail to reproduce the full features of correlation networks derived from MD. We further demonstrate that accurate prediction of residue level dynamic couplings requires a more detailed description of intra-protein interactions lacking in $\mathrm{Ca}$ based ENMs. Incorporating additional atomic details of both backbone and sidechain interactions is shown to yield significantly improved performance in terms of the reproduction of MD generated correlation networks. The predictive power of these more detailed ENMs is also illustrated by its applications to two additional allosteric protein systems, namely hemoglobin and caspase 7 . In both cases the model correctly identifies experimentally verified mutations that allosterically affect protein function. Importantly, despite the inclusion of additional backbone and sidechain atom details, atomistic ENM has a similar computational efficiency to conventional Ca based ENMs. This efficiency is orders of magnitude faster than conventional MD. Overall, our results indicate that ensemble based all-atom ENM derived network analysis can make allosteric predictions comparable to that based on MD and is thus a promising tool to more rapidly probe the allosteric features of biomolecular systems.

\section{Theoretical Methods}

All structure and ENM normal mode analysis were performed with Bio3D version 2.0 $0^{12-13}$. All-atom NMA was performed with Amber12 ${ }^{14}$. Molecular graphics were generated with 
VMD version $1.9^{15}$. Details about the structure preparation, principal component analysis, and MD simulations for $\mathrm{Ga}$ can be found in the ref. ${ }^{8}$.

\section{Atomistic elastic network model}

An atomistic elastic network model named 'AAENM' was developed, which has a similar energy function to conventional ENMs but has interaction sites defined by all the heavy atoms instead of just the $\mathrm{Ca}$ atoms. The energy function is defined by:

$$
U_{i j}(\mathrm{r})=k\left(\left\|\mathrm{r}_{i j}^{0}\right\|\right)\left(\left\|\mathrm{r}_{i j}\right\|-\left\|\mathrm{r}_{i j}^{0}\right\|\right)^{2}
$$

,where $\mathbf{r}$ is the current protein conformation, $\mathbf{r}^{\mathbf{0}}$ represents the equilibrium conformation, and $\left\|\mathbf{r}_{i j}\right\|$ the distance between atoms $i$ and $j$. The force constant $k$ is dependent on the equilibrium distance of two interacting atoms and has the functional form

$$
k(r)=\min \left\{7424 \cdot r^{-6}, 1500\right\}
$$

with unit of $k(r)$ given in $\mathrm{kcal}^{\mathrm{mol}}{ }^{-1} \AA^{-2}$. The parameters were obtained by fitting to the Amber 99SB force field as described in ref. ${ }^{16}$ (See also Figure S1). An all-atom model of crambin (PDB ID 3U7T) was prepared using the AmberTools package and minimized in NAB using conjugate gradient and Newton-Raphson until convergence. The Hessian matrix was extracted to obtain the force constants for all pair-wise heavy atoms. Fitting to the force constants was performed assuming an $r^{-6}$ decay ${ }^{16}$. The potential system dependency of the parameters in Eq. 2 was tested by performing similar all-atom Hessian calculations for three additional systems, namely ubiquitin, transducin, and the small GTPase Ras. Results indicate that the general trend of how all-atom force field derived effective force constants change with atom distance is very similar across these systems (Figure S1). This suggests that the current parameters are transferable to these and potentially other protein systems under model assumptions.

With Eq. (1), the Hessian matrix $\mathbf{K}$ of AAENM is a $3 M \times 3 M$ matrix, where $M$ is the number of heavy atoms. Therefore, direct normal mode analysis of AAENM generates normal modes with a dimension $3 \mathrm{M}$. In contrast, the normal modes of conventional $\mathrm{Ca}$ based ENMs have a dimension $3 N$, where $N$ is the number of residues. To facilitate quantitative comparisons between AAENM and conventional ENMs, an effective $3 N \times 3 N$ Hessian matrix $(\hat{\mathbf{K}})$ was calculated for AAENM as:

$$
\hat{\mathbf{K}}=\mathbf{K}_{\mathrm{AA}}-\mathbf{K}_{\mathrm{AQ}} K_{Q Q}^{-1} \mathbf{K}_{\mathrm{QA}}
$$

, where $\mathbf{K}_{A A}$ represents the sub-matrix of $\mathbf{K}$ corresponding to the $\mathrm{Ca}$ atoms, $\mathbf{K}_{Q Q}$ is the submatrix for the non-Ca atoms, and $\mathbf{K}_{A Q}$ and $\mathbf{K}_{Q A}$ are the cross sub-matrices between $\mathrm{Ca}$ and non-Ca ${ }^{16-17}$. Note that the actual $\hat{\mathbf{K}}$ used is in a mass weighted form $\mathbf{M}^{-1 / 2} \hat{\mathbf{K}} \mathbf{M}^{-1 / 2}$, where $\mathbf{M}^{-1 / 2}$ is a $3 N \times 3 N$ diagonal matrix containing the square root of residue masses (each 
residue mass is repeated three times for the $x, y$ and $z$ Cartesian coordinates). The normal modes and associated frequencies were then obtained by diagonalizing the (mass weighted)

$\hat{\mathbf{K}}$.

\section{Ensemble normal mode analysis}

The ensemble normal mode analysis approach applies NMA to all available crystallographic structures for a given protein family under study and facilitates the quantitative comparison of normal modes across heterogeneous structures (with unequal sequence composition and length). Here, we used the same Eq. (3) to calculate the effective Hessian matrix for all the aligned $\mathrm{Ca}$ atoms in the structural ensemble. In this case, $\mathbf{K}_{A A}$ in Eq. (3) represents the submatrix of the original Hessian matrix $\mathbf{K}$ corresponding to the aligned $\mathbf{C a}$ atoms, $\mathbf{K}_{Q Q}$ for the gapped regions, and $\mathbf{K}_{A Q}$ and $\mathbf{K}_{Q A}$ are the cross sub-matrices between the aligned and gapped regions ${ }^{16-17}$. Note that all normal mode calculations were performed on mass weighted effective Hessian matrices (See above).

\section{All-atom normal mode analysis}

All-atom NMA was performed with AMBER12 ${ }^{14}$ and corresponding force field ff99SB ${ }^{18}$ for all the 53 crystallographic structures of Ga. Nucleotide, ions, water molecules, and GDI were removed from the structure in advance (i.e. Ga was under the "apo" state during the calculation). Missing residues in structures were modeled with MODELLER 9.1 ${ }^{19}$. All nonstandard amino acid Mse were changed to the standard Met by replacing the selenium atom with the corresponding sulfur atom. In all systems, Arg and Lys were protonated while Asp and Glu were deprotonated. The protonation states for His residues were determined based on an inspection of the residues local environment and their pKa values as calculated by PROPKA ${ }^{20}$. The generalized Born (with dielectric constant 1.0) model $^{21}$ was employed to consider the solvation effect. Prior to NMA, 20000 steps of steepest descent followed by 50 steps Newton-Raphson energy minimization was performed.

\section{Mode filtering for the calculation of residue fluctuations and covariance}

The $3 N \times 3 N$ covariance matrix, $\Sigma^{(3 N)}$ for residue fluctuations is given by ${ }^{10}$

$$
\sum^{(3 N)}=k_{B} T \cdot \hat{\mathbf{K}}^{-1}
$$

,where $\hat{\mathbf{K}}^{-1}$ is the pseudo-inverse of the effective Hessian matrix $\hat{\mathbf{K}}^{\text {and is calculated by }}$

$$
\hat{\mathbf{K}}^{-1}=\sum_{i=1}^{P} \frac{1}{\lambda_{i}} \mathbf{u}_{i} \mathbf{u}_{i}^{T}
$$

,where $\mathbf{u}_{i}$ and $\lambda_{i}$ are the $i$ th (sorted in an increasing order of $\lambda$ ) eigenvector (the normal mode) and eigenvalue of $\hat{\mathbf{K}}$, respectively, and $P$ the number of modes included in the calculation. In normal cases, $P$ is equal to the degree of internal freedom, i.e. $3 N-6$ with $N$ the number of residues. In mode filtering, we considered a smaller value of $P$ to include only 
a certain number of the slowest modes. After obtaining the covariance matrix, residue fluctuations can be easily derived by summing up the diagonal entries of $\Sigma^{(3 N)}$ for each residue divided by residue mass.

\section{Correlation network analysis}

The linear Pearson inner product cross-correlation ${ }^{22}$ between residues $i$ and $j$ is calculated by

$$
c_{i j}=\frac{\operatorname{Tr}\left(\left[\sigma_{i j}\right]^{(3)}\right)}{\sqrt{\operatorname{Tr}\left(\left[\sigma_{i i}\right]^{(3)}\right)} \cdot \sqrt{\operatorname{Tr}\left(\left[\sigma_{j j}\right]^{(3)}\right)}}
$$

,where $\left[\sigma_{i j}\right]^{(3)}$ is the $3 \times 3$ cross sub-matrix of the covariance matrix $\Sigma^{(3 N)}$ between residues $i$ and $j,\left[\sigma_{i i}\right]^{(3)}$ (or $\left.\left[\sigma_{j j}\right]^{(3)}\right)$ the $3 \times 3$ diagonal sub-matrix for residue $i(j)$, and $\operatorname{Tr}(\cdot)$ is to take the trace of the matrix. A weighted network graph was constructed where each node represents an individual residue and the weight of the connection between nodes, $i$ and $j$, represents their respective cross-correlation value, $c_{i j}$. We used a network construction method similar to that introduced by Luthey-Schulten and colleagues ${ }^{3}$ and that we used previously for $\mathrm{Ga}$ MD based analysis ${ }^{8}$. Instead of two rules that were employed to build the network for MD, here only the second rule was adopted in the network construction for NMA: Network edges are added for residues satisfying $\left|c_{i j}\right| \geq_{O}$ in at least one structure and with a $\mathrm{Ca}$-Ca distance $d_{i j} \leq 10 \AA$ for at least $75 \%$ of total structures, where $c_{0}$ is a parameter to be determined (See below). The first rule in ref. ${ }^{8}$ to "save" constantly strong correlations across simulations between spatially distant residues was removed. This was based on the observation that not so many edges (a dozen versus more than one thousand total edges) were actually added through this rule for the MD simulations. Edge weights were then calculated as $-\log \left(\left|\left\langle c_{i j}\right\rangle\right|\right)$, where $\langle\cdot\rangle$ denotes the average across structures.

A model specific correlation threshold $c_{O}$ was employed. This was based on the observation that overall strength of correlation values generated from distinct methods can differ substantially. For example, the overall correlation values predicted by MD simulations are about two times higher than those predicted by ENMs (Figure S2). This indicates that using the same $c_{i j}$ threshold as that for MD with ENMs will lead to very sparse and possibly incomplete networks. To solve this problem, we determined $c_{0}$ for each individual method independently with the MD results for the GTP state as a reference. The objective here was to ensure all networks displayed a similar level of connectivity to their corresponding MD based networks. Specifically, we first identified that the threshold we used before, $c_{F}=0.6$ is equivalent to maximally keeping the top $\sim 3 \%$ correlations (the 97 th percentiles) from each correlation matrix to build the network for MD simulations under GTP (Fig. S2). The maximal value of the 97th percentiles in the correlation matrices derived from e.g. HCA (the ENM proposed by Hinsen et al. ${ }^{16}$ ) for the same state is 0.3 . $c_{0}$ was thus set to be 0.3 for HCA. Similar algorithms were employed to determine the thresholds for other methods. 
The community, centrality, and suboptimal path analysis for NMA networks are essentially the same as those in the analysis of MD simulations. Here we give a brief description of the algorithms and details can be found in ref. ${ }^{8}$. The same definition of communities as in MD studies was adopted to facilitate a direct comparison between NMA and MD. These communities were derived by first doing a betweenness clustering algorithm similar to that introduced by Girvan and Newman ${ }^{23}$ and then taking a consensus to capture the features that the original community partitions by clustering are largely consistent across states except for some variations localized to the nucleotide binding regions. Inter-community correlations were then calculated as the sum of the mean correlation values across structures associated with all the inter-community edges. A standard $t$-test was also performed to measure the significance of the mean difference between inter-community correlations of distinct states. Node centralities that assess the density of connections per node were calculated by solving the eigenvalue problem of the adjacent matrix A. Node centralities were defined by the entries in the first (with the largest eigenvalue) eigenvector. All centralities were also scaled by the largest centrality value. Otherwise indicated, 500 paths were collected for each source/sink pair in the path analysis. Comparative path length distributions indicating the strength of correlated motions under distinct conditions were also calculated. In addition, normalized node degeneracy, i.e. the fraction of the number of paths going through each node, was calculated.

\section{PCA for the correlation matrices derived from ensemble NMA of Ga, hemoglobin, and caspase 7 crystallographic structures}

PCA was applied to the residue cross-correlation matrices derived from ensemble NMA. Singular value decomposition was performed on the matrix in which each row containing the upper-triangular entries of the correlation matrix derived from each structure. The obtained eigenvector with the largest eigenvalue indicates key residue couplings (corresponding to the positions with high absolute values in the eigenvector) that distinguish states (e.g. Figure 1C).

\section{Similarity measurement}

Root mean square inner product (RMSIP)—Root mean square inner product (RMSIP) measures the cumulative overlap between all pairs of the 1 largest eigenvectors ${ }^{24}$, and is defined as:

$$
R M S I P=\left[\frac{1}{l} \sum_{i=1}^{l} \sum_{j=1}^{l}\left(v_{i}^{A} \cdot v_{j}^{B}\right)^{2}\right]^{\frac{1}{2}}
$$

, where $\mathbf{v}_{1}^{\mathrm{A}}$ and $\mathbf{v}_{1}^{\mathrm{B}}$ represent the $\dot{t}$ th and $j$ th eigenvectors obtained from protein $\mathrm{A}$ and $\mathrm{B}$, respectively, $I$ is the number of modes to consider which is commonly chosen to be 10 . The RMSIP measure varies between 0 (orthogonal) and 1 (identical directionality). 
The Bhattacharyya's coefficient (BC) ${ }^{11}$ was employed to measure the similarity between two covariance matrices. Before the calculation, both covariance matrices were normalized by the matrix trace. The coefficient can be written as

$$
B C=\exp \left[-\frac{1}{2 q} \ln \left(\frac{|\mathbf{\Lambda}|}{\left(\left|\mathbf{Q}^{\mathrm{T}} \sum_{\mathrm{A}} \mathbf{Q}\right|\left|\mathbf{Q}^{\mathrm{T}} \sum_{\mathrm{B}} \mathbf{Q}\right|\right)^{1 / 2}}\right)\right]
$$

,where $\mathbf{Q}$ is a matrix in which columns are eigenvectors of the averaged covariance matrix $\left(\Sigma_{\mathrm{A}}+\Sigma_{\mathrm{B}}\right) / 2, \Lambda$ is a diagonal matrix containing the corresponding eigenvalues, and $q$ the number of modes needed to capture $90 \%$ of the variance (cumulative eigenvalues) of $\mathbf{Q}$. The $\mathrm{BC}$ varies between 0 and 1 , and equals to 1 if the covariance matrices $\left(\Sigma_{\mathrm{A}}\right.$ and $\left.\Sigma_{\mathrm{B}}\right)$ are identical.

Squared inner product (SIP) measures the linear correlation between two vectors, $\boldsymbol{w}_{\boldsymbol{A}}$ and $W_{B}$, and it was applied to compare atomic fluctuation profiles, inter-community edge weights, node centrality profiles, and node degeneracy profiles. The score also varies between 0 and 1 and is defined as

$$
S I P=\frac{\left(w_{A}^{T} w_{B}\right)^{2}}{\left(w_{A}^{T} w_{A}\right)\left(w_{B}^{T} w_{B}\right)}
$$

\section{Structure preparation, principal component analysis, ensemble normal mode analysis, and correlation network analysis for hemoglobin and caspase 7}

Collection of structures for hemoglobin and caspase 7 were seeded from a chain based BLAST against the RCSB PDB database with the query sequence PDB code '2DN1' and ' $1 \mathrm{~F} 1 \mathrm{~J}$ ', respectively. By this, 329 and 9 crystallographic structures were obtained for hemoglobin and caspase 7, respectively, after removing structures with long consecutive missing residues. All structures were then reconstructed to their canonical oligomeric forms, i.e. heterotetramer $(\mathrm{a} 1 / \beta 1 / \mathrm{a} 2 / \beta 2)$ for hemoglobin and homodimer for caspase 7 , based on the crystal symmetry using the Bio3D package. Prior to further analysis, the structurally invariant 'core' residues were identified as previously described ${ }^{25}$ and used for superposition of all crystal structures. PCA was used to characterize inter-conformer relationships. Briefly, PCA diagonalizes the positional variance-covariance matrix, $\Sigma$, with elements $\Sigma_{\mathrm{ij}}$ calculated from the Cartesian coordinates of equivalent superposed $\mathrm{Ca}$ atoms in all structures. The eigenvectors, or principal components (PCs), of $\Sigma$ are linear combinations of the Cartesian axes and form a set of new axes along which the structural variance is largest. Typically, only a few PCs with the largest eigenvalues form a subspace that can explain most of the variability in the original structures. 
The same ensemble NMA and correlation network analysis to those used for Ga (See above) were performed for hemoglobin and caspase 7 . In the path analysis, multiple source/ sink pairs that constitute the binding sites of the substrates and the effectors were adopted, to account for the possible multiple routes of allosteric regulation in hemoglobin and caspase 7. For each pair, 100 suboptimal paths were computed, after which a path length weighted normalized node degeneracy was calculated.

\section{Random model used to calculate the significance of predictions for hemoglobin and caspase 7}

For a system with $N$ residues and $L$ experimentally verified positive mutations, the significance of a prediction that identifies $l$ experimentally verified key residues in total $M$ predicted key residues was defined by the probability of randomly choosing $M$ residues that has the same or larger hit rate:

$$
P(\text { Hit } \geq l \mid M, L, N ; \text { random })=\sum_{\mathrm{i}=1}^{\min (\mathrm{L}, \mathrm{M})} \frac{1}{\mathrm{C}_{\mathrm{N}}^{\mathrm{M}}} \cdot \mathrm{C}_{\mathrm{L}}^{\mathrm{i}} \cdot \mathrm{C}_{\mathrm{N}-\mathrm{L}}^{\mathrm{M}-\mathrm{i}}
$$

\section{Results and Discussion}

\section{Ensemble NMA predicts distinct global and local dynamics for GTP, GDP and GDI forms of $\mathrm{Ga}$ that are consistent with MD}

Applying elastic network model normal mode analysis to the 53 available $\mathrm{Ga}$ crystallographic structures (Table S1) predicts ligand dependent modes of motion.

Clustering based on the root mean square inner product (RMSIP) of the calculated modes of all structures clearly distinguishes GTP, GDP and GDI (GDP dissociation inhibitor) associated states (Figure 1A). This partitioning is consistent with Cartesian coordinate PCA of this same structure set (see Figure S3 and ref. ${ }^{8}$ ). The GDI state structures display the most distinct modes. These include the opening-closing of the switch regions towards the P-loop (PL) (Figure S4), which in turn accounts for the comparatively large predicted flexibilities for these regions in all GDI structures (Figure 1B). These NMA derived residue fluctuations also clearly distinguish states revealing areas of conserved dynamics interspersed with regions of significantly distinct flexibilities in each state (Figure 1B). Specifically, the PL, SI, SII and SIII (switch regions I-III) are significantly more flexible in GDP and GDI states than that in the GTP state (with a $p$-value $<0.05$ ). These results indicate that stabilization of the P-loop and switch regions is indicative of the active state and suggest that this relative stabilization may be a prerequisite for binding of effectors and regulatory proteins that are known to preferentially bind the 'active' GTP state. This finding is consistent with that derived from extensive (fifteen 80-ns) MD simulations (Figure S5). These MD simulations also reveal additional dynamic difference including a more flexible loop 7 (between a 3 and $\beta 5$ ) in the GTP state and a broader extent of the helical domain (HD) regions with distinct flexibilities (Figure S5). 
NMA reveals state specific residue-residue dynamic couplings-Performing PCA on the residue-residue couplings obtained from NMA on all structures (see Methods) reveals that the first principal component (PC1) clearly separates GTP, GDP and GDI states (Figure 1C). This is in contrast to standard pairwise RMSD clustering that does not clearly separate states (Figure S3), or indeed coordinate based PCA, which requires at least two PCs to distinguish states to a somewhat lesser degree than NMA correlation data (e.g. the clear separation for Gi and Gt GTP forms with NMA, see Figure S6 for comparison). Further analyzing the contributions to coupling based $\mathrm{PC} 1$ reveals individual residue-residue couplings that collectively distinguish states. Consistent with MD simulations ${ }^{8}$, this analysis indicates that the GTP state has stronger couplings in the functionally critical P-loop and switch regions whereas the GDI state has uniquely strong couplings around the region between the Ras-like domain (termed RasD) and HD. Intriguingly, NMA also reveals that the GDI state has the strongest coupling values around the region between loop $\mathrm{C}(\mathrm{aB} / \mathrm{aC})$ and loop F (aE/aF) (Figure 1D). This novel result from NMA was not apparent from extensive MD where the coupling values for this region were observed to be similar across states (Figure $2 \mathbf{c}$ in ref. ${ }^{8}$ ). In summary, performing NMA across the ensemble of available $\mathrm{Ga}$ structures captures important information on state specific global and local dynamics of potential functional relevance that is also observed in more expensive MD simulations. It is important to note in this regard that comparing any single structure NMA results (single GTP $v s$ single GDI NMA for example) does not robustly reveal the same detailed state specific trends as our multiple MD or considerably less expensive multi-structure NMA approach.

\section{Conventional ENM approaches have limited ability to reproduce the correlation network analysis results generated from MD}

Ensemble NMA based on the conventional ENM (the harmonic Ca potential model, or HCA as originally proposed by Hinsen ${ }^{16}$ ) generates state specific networks of residue correlations that only partially agree with those from MD simulations. Correlation networks were built with an ensemble averaged algorithm similar to that used previously for MD simulations (See ref. ${ }^{8}$ and Methods). In contrast to the eight peaks apparent in the centrality analysis of MD networks, NMA based networks depict only five prominent centrality peaks located in the lobe-2 region and the HD (Figure S7). The main difference lies in the lobe-1 region of the GTP state (with a similarity score of only 0.03; See Methods for more details). Most notably the three main centrality peaks around the PL, SI and SII regions obtained from MD are absent from the NMA generated networks. Also in contrast to MD results the centrality values for the lobe-2 region of the NMA GTP network are indistinguishable from that of the GDP and GDI networks. Similarly, the GTP state community analysis results differ between MD and NMA (Figure S8). In particular, the relative coupling strength between PL, SI and SII is substantially reduced in the NMA results, although it is still stronger than those obtained for GDP and GDI associated conditions. Moreover, several notable GTP specific inter-lobe couplings observed in MD simulations, including SIII/PL, SIII/C1 and PL/C2 are reduced or absent from the NMA results. Intriguingly, most of the GDP featured couplings located in the lobe- 2 region and the GDI featured inter-domain coupling between HD2 and SIII are captured by the NMA method. 
Network path analysis results also differ between MD and NMA. Between two distal functional sites (corresponding to receptor binding site and domain-domain interface, respectively), 500 optimal (shortest) and suboptimal (close to but longer than the optimal) paths were explored. This analysis reveals potential pathways of residues that mediate the coupling between receptor binding and domain opening required for $\mathrm{G}$ protein activation ${ }^{8}$. NMA GDI state network results have nearly no overlap with those in the corresponding MD generated network (Figure S9). In addition, path length distributions differ substantially between MD to NMA results. MD paths in the GTP network are much shorter than those in the GDP and GDI networks, while for NMA paths are nearly indistinguishable across states. Together with the centrality and community analysis, our results indicate that in spite of the overall good prediction of local and global dynamics, ensemble NMA based on the conventional ENM approach cannot fully reproduce the results obtained from MD based correlation network analysis. The latter is expected to be more dependent on the precise prediction of the residue-residue covariance/correlations, a feature apparently lacking in current state of art ENMs. In the following sections, we extend our evaluation to a broader set of ENM approaches to explore the factors affecting correlation network prediction accuracy in relation to MD results.

\section{Benchmark of popular ENM approaches}

In this section we evaluate the performance of nine different ENM approaches in relation to extensive Ga MD simulations (fifteen $80-n s)^{8}$ (see Table 1). This includes HCA $^{16}$, the anisotropic network model (ANM) ${ }^{26}$, the Gaussian network model (GNM) ${ }^{27}$ and the sequence- and distance-dependent model (sdENM) ${ }^{28}$. For ANM, two cutoff values were tested, 8.0 $\left(\mathrm{ANM}_{0.8}\right)$ and $18.0 \AA\left(\mathrm{ANM}_{1.8}\right)$, to evaluate how the range of included interactions affect model predictions. Two modified HCA variants are also included in the comparison set: 'varHCA' has force constants scaled by atomic structural variance derived from crystal structures ${ }^{13}$, and ' $m f H C A$ ' (short for 'mode filtered' HCA) has the same energy function as HCA but omits high frequency modes in generating fluctuation and covariance data (See Methods). To further investigate how the atomic details of protein backbone and side chains affect model performance, we introduce an atomistic ENM where nodes represent all protein heavy atoms ('AAENM'; See Methods). Similarly, a 'mode filtered' version AAENM ('mfAAENM') is included to evaluate the effect of removing fast modes from this model. Performance is evaluated with multiple metrics including conventional square inner product (SIP) of normalized atomic fluctuations and the Bhattacharyya's coefficient $(\mathrm{BC})$ of trace-normalized covariance ${ }^{11}$. We also introduce a number of metrics relevant to correlation network analysis. This includes the SIP of the pre-ordered list of edge weights between network communities ('Community'), the SIP of node centrality profiles ('Centrality') and the SIP of node degeneracy profiles in ( $k$-shortest) suboptimal paths ('Path') between previously defined functional sites (termed source and sink). Here we utilized the same source/sink pair (K31 on $\beta 1$ and D146 on the interface of the helical domain and the Ras-like domain) relevant to the receptor catalyzed $\mathrm{Ga}$ activation as that used in previous work ${ }^{8}$. All calculations were performed under GTP, GDP and GDI states. For comparison, we also included normal mode analysis results obtained with the all-atom Amber force field ('AmberNMA') and benchmarked all models against the atomic 
fluctuations derived from multiple crystal structures ('X-ray') and their $\mathrm{Ca}$ atom B-factors ('B-factor').

Atomic fluctuation, residue covariance and residue correlation network results are consistent across independent MD simulations. As the "gold standard" in the benchmark, results from MD simulations are expected to be robust and independent of initial simulation conditions. To test this assumption, we performed an independent set of fifteen 80-ns simulations, five for each GTP, GDP and GDI state. Results for fluctuation and covariance analysis yield high similarity scores $(>0.6)$ even between single simulation trajectories arbitrarily chosen from the two simulation sets (Figure 2A-C). With averaging over multiple simulation trajectories, both scores increase monotonically and reach $>0.8$ for all three states when five trajectories are included. Correlation network analysis results are also consistent $(>0.7)$ across distinct initial simulation conditions when all the five trajectories are considered, whereas scores vary from near 0 to over 0.9 when less than five trajectories are used. In summary, MD simulations are generally robust in terms of the metrics considered in this work and can be used as a reliable standard for assessing the performance of different ENM approaches.

\section{Ensemble NMA has improved performance over single structure NMA-NMA}

characterizes protein dynamics proximal to a local energy minimum. This implies that predictions from NMA approaches can be dependent on the (often arbitrarily chosen) initial structure used for calculations. NMA applied to a single structure can thus be strongly biased to the specific state represented by the structure and may fail to capture the more global picture of structural dynamics ${ }^{29}$. This input structure dependency of NMA also provides an effective way to characterize and potentially classify protein structures via a comparison of NMA derived dynamics. Using this approach additional structural dynamic insight can be obtained that is not readily available from conventional structural comparison. This realization led to the development of the ensemble NMA approach, which calculates modes for all structures in a systematic and unified manner. The subsequent clustering based on the calculated modes and characterization of each cluster (or state) can then effectively reveal the dynamical heterogeneity that is missing in single structure based NMA (See e.g. Figure 1A-D). Indeed, detailed comparison shows that ensemble NMA has an overall improvement over single structure NMA in predicting many properties of Ga structural dynamics (See Figure 3A-C, Table S2 \& S3). In terms of fluctuations and covariance estimates, ensemble averaged results are generally better than those from single structure NMA for seven distinct methods, i.e. mfAAENM, mfHCA, AAENM, AmberNMA, $\mathrm{ANM}_{1.8}, \mathrm{HCA}$, and sdENM (Figure 3A-C). A more pronounced improvement is evident for the prediction of network properties. In particular, the predicted suboptimal paths for the GTP state network using ensemble based AAENM and AmberNMA have a similarity score (SIP) $>0.3$ higher than those predicted by corresponding single structure NMA (with a total score of 0.92 and 0.78 respectively).

The conformational diversity in the input structure set underlies the superior performance of ensemble NMA over single structure NMA. We examined the effect of this diversity on predictive performance by consecutively removing structures in a random fashion from the input ensemble (Figure S10). The predictive power decreases significantly for all metrics when there is no representative structure remaining for one or more of the major GTP, GDP, 
or GDI states. The decreased performance is most notable for network analysis related metrics (a decrease of 0.12 from 0.74 for sub optimal path, 0.57 to 0.8 for centrality and 0.72 to 0.84 for community scores). Note that ensemble NMA performed with one structure per state (dashed lines in Figure S10) has overall lower prediction scores than ensemble NMA using more than one structures per state. This indicates that the comparatively small structural variance within each state also contributes to the improved performance of the ensemble NMA approach. Unless otherwise indicated, all subsequent ENMs discussed in this work are implemented using ensemble NMA with all available crystallographic structures included.

Most ensemble ENM methods tested can reproduce fluctuation and covariance results obtained from MD simulations. Typical scores range from 0.7 to 0.9 for fluctuations and from 0.85 to 0.9 for covariance (Table 2). These scores are close to those obtained for ensemble AmberNMA and control MD simulation sets ( 0.87 and 0.92 respectively). Our results of comparing atomic fluctuations and covariance are overall consistent to previous work by Fuglebakk et al. ${ }^{11}$ (Table $2 \& \mathrm{~S} 4$ ). In particular, we found that $\mathrm{ANM}_{1.8}$ has the lowest scores $(<0.70)$ for the prediction of covariance estimates. This indicates that models with a long range cutoff may have imposed too many spatial constraints to accurately reproduce collective motions. Indeed, with a shorter range cutoff $\mathrm{ANM}_{0.8}$ yields improved covariance prediction with Bhattacharyya coefficient (BC) scores $>0.85$ (Table 2). In contrast, $\mathrm{ANM}_{1.8}$ has very high SIP scores ( 0.9) for fluctuations when B-factors are used for comparison (Table S4). Interestingly, in the same benchmark against B-factors, MD yields lower scores ranging from 0.74 to 0.87 . This implies that B-factors may partially reflect thermal motions in a spatially constrained crystal environment rather than the more mobile solution environment. As discussed in Fuglebakk et al. ${ }^{11}$, B-factors may not be an ideal standard to benchmark prediction of protein motions in solution. We also note that $\mathrm{ANM}_{0.8}$ has very low scores $(0.21 \sim 0.65)$ for the prediction of fluctuations (Table 2). Detailed analysis indicates that a few highly mobile residues with minimal restraints underlie the poor performance of $\mathrm{ANM}_{0.8}$. For example, with $\mathrm{ANM}_{0.8}$ in one of the structures under the GDP state (PDB ID: 1TAG) T177 in the SI region is predicted to have orders of magnitude larger fluctuation than other residues. After normalization, the entire fluctuation profile is drastically changed due to this single peak value leading to a low overall SIP score. Similar phenomena for short-range cutoff ANM has been described previously ${ }^{11}$.

Fluctuations predicted by ENMs, AmberNMA and MD display only moderate similarity to that determined directly from available crystallographic structures. This indicates that the crystallographic data set contains a degree of structural heterogeneity that is not captured by current state of art computational methods (Table S4). The best SIP score of fluctuations across all methods and all states benchmarked with the crystallographic structural variance is 0.58 . For most methods, the scores are below 0.5 . The major reason of such difference is found to be the underestimated flexibility of the switch regions in computational models. For example, in both GDP and GDI states, crystallographic structures reveal that the SII region has much higher fluctuations than remaining residues, whereas such differences are substantially reduced in predictions from both ENMs and MD. As expected, taking into 
account the information from experimental data (leading to the varHCA model) significantly improves the prediction of crystallographically revealed structural variance (Table S4).

Suboptimal path prediction improves with atomistic ENM (AAENM)-Most conventional $\mathrm{Ca}$ based ENMs (HCA, GNM, sdENM, and ANMs) cannot fully reproduce correlation network analysis results derived from MD (Table 3). For the GTP state scores range from 0.3 to 0.6 for network community analysis, from 0.3 to 0.35 for network centrality, and 0.1 to 0.4 for network path analysis. The variable spring constant approach varHCA has slightly improved prediction for both community and path analysis but no significant improvement for centrality estimates. Intriguingly, atomistic AAENM has significantly improved prediction for network paths in the GTP network (with the score 0.92; See Table 3). This indicates that the additional structural detail included in the AAENM approach is important for accurate prediction of critical couplings of distal functional sites. This is supported by the observation that the energetically more precise AmberNMA gives similar high level of prediction for paths in the GTP network (SIP 0.78). It is also important to note that sdENM, which incorporates amino acid specific force constants, has very low scores for GTP state path analysis (0.06). This indicates that simply factoring amino acid sequence information without their additional atomistic details does not improve path analysis predictions.

Excluding the fastest modes from AAENM further improves model performance, leading to the best performance (mfAAENM) in the current benchmark. In particular, prediction of network node centrality under the GTP state is poor for all the simplified methods without mode filtering including AmberNMA, with similarity scores generally lower than 0.35 (Table 3). Detailed inspection shows that, in MD, the functional regions coordinating the phosphate of the nucleotide, including PL, SI and SII are highly coupled, leading to peaks around these regions in the node centrality profile (Figure S7). These couplings near the nucleotide binding sites are substantially reduced in ENMs and AmberNMA, possibly due to the underestimation of collective motion. Explicitly including the nucleotide in the energy function does not substantially improve performance, since a modified AAENM considering both nucleotide and magnesium ion yields similar predictions (with a centrality SIP score of 0.28). In contrast, filtering of the fastest modes in both HCA and AAENM substantially improves prediction (with centrality scores rising to $>0.7$, see Figure $4 A \& B$ ). In terms of the centrality score, the optimal number of slowest modes that should be retained in the calculation is found to be near 10 for both HCA and AAENM. Similar high level prediction (>0.6) is retained in AAENM with the number of slowest modes included raised up to 400, whereas the score drops to near 0.5 rapidly after using more than 10 slowest modes in HCA. Modes in the moderate frequency range are also likely to be informative, as mfHCA in which only the 10 slowest modes are retained displays relatively low covariance scores in relation to MD (Table 2). Intriguingly, mfAAENM (with 400 slowest modes) also shows the best prediction scores for many other comparisons such as network communities and ranks high in most of remaining metrics with the only exception being the prediction for paths under the GDI state (Table $2 \&$ 3). In particular, previously revealed residues (L32, R174, and T177) with MD and experimental mutagenesis that are critical to $\mathrm{Ga}$ activation ${ }^{8,30-31}$ are also identified as a notable "on path" residue in path analysis using mfAAENM, 
AAENM, and AmberNMA under the GTP state. This result indicates that these models are indeed suitable for the analysis of $\mathrm{G}$ protein allosteric mechanisms. In subsequent sections we expand analysis to consider additional protein families.

\section{Inference of allosteric networks in hemoglobin and caspase 7 with ensemble NMA}

Ensemble NMA reveals local and global dynamic patterns that distinguish the oxygen binding states of hemoglobin. Hemoglobin is the vital molecule in red blood cells responsible for transporting oxygen from the lungs to other tissues. Functional hemoglobin forms a heterotetramer $\left([a \beta]_{2}\right)$ with each subunit binding one oxygen molecule (Figure $5 \mathrm{~A}$ ). The interplay between subunits results in an apparent cooperativity of oxygen binding, which makes hemoglobin a prototype system for studying protein allostery. We collected a total of 329 available crystallographic structures of the hemoglobin tetramer and dimer from RCSB PDB database. All dimers were reconstructed into the canonical tetramer form based on crystal symmetry. Ensemble NMA using the conventional HCA model reveals two major groups of structures with distinct predicted dynamics (Figure 6A). These groups coincide with the two major conformational clusters, "relaxed" (R) and "tense" (T) states of oxygen binding, derived from a principal component analysis of superposed structures (Figure S11). Ensemble NMA results indicate that the dynamic difference between $\mathrm{R}$ and $\mathrm{T}$ states is largely localized to the subunit-subunit interfaces (Figure 6B-D). In particular, residues at the $a_{1} / \beta_{2}$ and $\beta_{1} / a_{2}$ interfaces display higher flexibility in the $R$ state whereas those at the $a_{1} / a_{2}$ interface show higher flexibility in the $T$ state. The overall dynamic residue crosscorrelations at these interfaces are substantially enhanced in the $\mathrm{T}$ state exhibiting an effective "dynamical tightening" (Figure 6D).

Prediction of allosteric networks in hemoglobin and the identification of key residue perturbations that may affect cooperative oxygen binding are overall consistent with experimental data. Thirty-eight representative tense state structures (after removing redundant structures with RMSD less than $0.5 \AA$ to any other representative structures) were subjected to network analysis using both conventional (HCA) and newly developed ENMs (AAENM and mfAAENM). For comparison, we also included a model that employs a simple structural contact map to build a structure only network (i.e. omitting dynamic details). Key residues were identified as those with high node degeneracy ( $\searrow 0.3)$ in a path analysis, in which source and sink are chosen as oxygen binding sites in each subunit. Instead of using a single pair of source/sink, here we performed path analysis for every pair of oxygen binding site residues (100 paths for each pair). Normalized node degeneracy was then calculated from the cumulative suboptimal paths, with each path weighted by the path length. Previously published experimental mutation data were used to assess the accuracy of predicted key residues. In particular, we used the mutations that most likely occur within (positive) or out of (negative) the allosteric network (See Figure 5A). This analysis indicates that key residues predicted by each model only represent a small subset of the entire protein $(<15 \%)$ (Table 4). In particular, mfAAENM identifies 67 ( 12\% of the total 547 residues) key residues, four of which occur in the positive experimental dataset. Two additional experimentally verified allosteric mutants are missed by the model. Detailed inspection shows that these two residues are located at the protein surface in contrast to the other six sites. The hit rate of mfAAENM for experimental results is also significant ( $p$-value 0.003 ) 
with respect to a random model (See Methods). In contrast, only two positive experimental mutations are identified by HCA and AAENM methods. Note that neither of the two experimentally verified negative mutations (i.e. have no effect to the oxygen binding kinetics) is in the set of predicted key residues for any of the methods. Importantly, the structure only network method, that does not include predicted dynamics, does not identify any of the positive experimental mutations in its predicted set of 44 residue ( $\sim 8 \%$ of total residues). This result highlights the importance of including dynamic information into network analysis for the study of protein allosteric mechanisms.

Ensemble NMA predicts active and inactive state specific dynamics of caspase 7. Caspases, or cysteine aspartate proteases, are a large family of proteases playing an important role in apoptosis. Caspase 7 is the most well characterized family member and serves as a useful representative for investigating the structure and dynamics of the caspase family as a whole. We collected a total of nine crystallographic structures of caspase 7 from the PDB. Monomeric structures were reconstructed into homodimers based on annotated crystal symmetry. PCA of the caspase 7 structures reveals a major conformational cluster representing the active state and also identifies two distinct structures representing the intermediate and inactive states respectively (Figure S12). Ensemble NMA reveals an apparent dynamic difference between active, intermediate, and inactive states consistent with coordinate PCA (Figure 7A-D). In particular, the functional loops L2, L3 and L4 that are critical for substrate binding and catalysis are predicted to be much more flexible in the inactive and intermediate states, whereas these loops are largely stabilized in the active state (Figure 7B). Cross-correlation analysis further reveals that these functional loops together with L2' and the loops containing catalytic sites (the Cys-His dyad) are more strongly coupled in the active state than those in the inactive and intermediate states (Figure 7C\&D). These stabilization and coupling effects could be critical for the activation of caspase 7 and subsequent substrate binding and catalysis.

Similar performance of ENMs on the prediction of allosteric network residues is observed for caspase 7. With the seven active form structures, ensemble NMA based network analysis was applied to infer the allosteric networks that potentially link between the Cys-His dyad catalytic sites on one chain and the C-terminal L2' on the other chain (See Figure 5B). Again the mfAAENM is shown to be the best performing model correctly capturing four out of six experimentally verified positive mutations in a set of 36 highlighted residues ( $>8 \%$ of the total 459 residues, Table 4). The significance values for mfAAENM, AAENM, and HCA are all $<0.1$, with mfAAENM showing the highest significance $(<0.001)$. The structure only network approach performs the worst, capturing only two of the experiment mutations with a significance value $>0.1$.

\section{Conclusions}

Most of the nine NMA approaches analyzed (Table 1) did remarkably well at predicting fluctuations and covariance. Exceptions were ANM with either too long or too short interaction range and mfHCA in which too many modes of moderate frequency are excluded. Overall the mfAAENM approach that incorporates interaction details of all heavy atoms and excludes modes in the high frequency range outperformed other methods tested. 
Improvements were minor but significant for fluctuations and covariance and particularly noteworthy for dynamic residue cross-correlations and subsequent network analysis. In addition, utilizing an ensemble approach, where calculations are applied to all available structures, improves dynamic predictions of all methods tested and can yield information on functional state specific global and local dynamics that are consistent with MD simulations. We note that all methods tested in this work are made freely available within the Bio3D package for structural bioinformatics ${ }^{12-13}$. Furthermore, we are in the process of developing the eNMA approach as an interactive online application currently available from: http:// thegrantlab.org/bio3d/webapps. Collectively, our results indicate that ensemble NMA can be a promising tool for rapidly predicting protein structural dynamics and allosteric sites.

\title{
Supplementary Material
}

Refer to Web version on PubMed Central for supplementary material.

\section{Acknowledgments}

We thank Drs. G. Scarabelli and J. Tesmer for valuable discussions. We acknowledge support from the University of Michigan and the National Institute of Health (NIH: 2R01GM070862-10A1).

\author{
ABBREVIATIONS \\ PCA principal component analysis \\ MD molecular dynamics \\ NMA normal mode analysis \\ ENM elastic network model \\ GDI GDP dissociation inhibitor \\ RasD Ras-like domain \\ HD helical domain
}

\section{References}

1. Goodey NM, Benkovic SJ. Allosteric Regulation and Catalysis Emerge Via a Common Route. Nat Chem Biol. 2008; 4:474-482. [PubMed: 18641628]

2. Nussinov R, Tsai C-J. Allostery in Disease and in Drug Discovery. Cell. 2013; 153:293-305. [PubMed: 23582321]

3. Sethi A, Eargle J, Black AA, Luthey-Schulten Z. Dynamical Networks in Trna:Protein Complexes. Proc Natl Acad Sci U S A. 2009; 106:6620-6625. [PubMed: 19351898]

4. Gasper PM, Fuglestad B, Komives EA, Markwick PRL, McCammon JA. Allosteric Networks in Thrombin Distinguish Procoagulant Vs. Anticoagulant Activities. Proc Natl Acad Sci U S A. 2012; 109:21216-21222. [PubMed: 23197839]

5. Vanwart AT, Eargle J, Luthey-Schulten Z, Amaro RE. Exploring Residue Component Contributions to Dynamical Network Models of Allostery. J Chem Theory Comput. 2012; 8:2949-2961.

[PubMed: 23139645] 
6. Scarabelli G, Grant BJ. Kinesin-5 Allosteric Inhibitors Uncouple the Dynamics of Nucleotide, Microtubule, and Neck-Linker Binding Sites. Biophys J. 2014; 107:2204-2213. [PubMed: 25418105]

7. Guo J, Pang X, Zhou H-X. Two Pathways Mediate Interdomain Allosteric Regulation in Pin1. Structure. 2015; 23:237-247. [PubMed: 25543254]

8. Yao XQ, Malik RU, Griggs NW, Skjaerven L, Traynor JR, Sivaramakrishnan S, Grant BJ. Dynamic Coupling and Allosteric Networks in the Alpha Subunit of Heterotrimeric G Proteins. J Biol Chem. 2016; 291:4742-4753. [PubMed: 26703464]

9. Feher VA, Durrant JD, Van Wart AT, Amaro RE. Computational Approaches to Mapping Allosteric Pathways. Curr Opin Struct Biol. 2014; 25:98-103. [PubMed: 24667124]

10. Bahar I, Lezon TR, Yang LW, Eyal E. Global Dynamics of Proteins: Bridging between Structure and function. Annu Rev Biophys. 2010; 39:23-42. [PubMed: 20192781]

11. Fuglebakk E, Reuter N, Hinsen K. Evaluation of Protein Elastic Network Models Based on an Analysis of Collective Motions. J Chem Theory Comput. 2013; 9:5618-5628. [PubMed: 26592296]

12. Grant BJ, Rodrigues AP, ElSawy KM, McCammon JA, Caves LS. Bio3d: An R Package for the Comparative Analysis of Protein Structures. Bioinformatics. 2006; 22:2695-2696. [PubMed: 16940322]

13. Skjaerven L, Yao XQ, Scarabelli G, Grant BJ. Integrating Protein Structural Dynamics and Evolutionary Analysis with Bio3d. BMC Bioinformatics. 2014; 15:399. [PubMed: 25491031]

14. Case, DA., Darden, TA., Cheatham, TEIII., Simmerling, CL., Wang, J., Duke, RE., Luo, R., Walker, RC., Zhang, W., Merz, KM., et al. Amber 12. University of California; San Francisco: 2012.

15. Humphrey W, Dalke A, Schulten K. Vmd: Visual Molecular Dynamics. J Mol Graph. 1996; 14:3338. [PubMed: 8744570]

16. Hinsen K, Petrescu A-J, Dellerue S, Bellissent-Funel M-C, Kneller GR. Harmonicity in Slow Protein Dynamics. Chem Phys. 2000; 261:25-37.

17. Fuglebakk E, Echave J, Reuter N. Measuring and Comparing Structural Fluctuation Patterns in Large Protein Datasets. Bioinformatics. 2012; 28:2431-2440. [PubMed: 22796957]

18. Hornak V, Abel R, Okur A, Strockbine B, Roitberg A, Simmerling C. Comparison of Multiple Amber Force Fields and Development of Improved Protein Backbone Parameters. Proteins. 2006; 65:712-725. [PubMed: 16981200]

19. Eswar N, Webb B, Marti-Renom MA, Madhusudhan MS, Eramian D, Shen MY, Pieper U, Sali A. Comparative Protein Structure Modeling Using Modeller. Curr Protoc Protein Sci. 2007 Chapter 2, Unit 2.9.

20. Li H, Robertson AD, Jensen JH. Very Fast Empirical Prediction and Rationalization of Protein Pka Values. Proteins. 2005; 61:704-721. [PubMed: 16231289]

21. Tsui V, Case DA. Theory and Applications of the Generalized Born Solvation Model in Macromolecular Simulations. Biopolymers. 2000; 56:275-291.

22. Ichiye T, Karplus M. Collective Motions in Proteins: A Covariance Analysis of Atomic Fluctuations in Molecular Dynamics and Normal Mode Simulations. Proteins. 1991; 11:205-217. [PubMed: 1749773]

23. Girvan M, Newman ME. Community Structure in Social and Biological Networks. Proc Natl Acad Sci U S A. 2002; 99:7821-7826. [PubMed: 12060727]

24. Amadei A, Ceruso MA, Di Nola A. On the Convergence of the Conformational Coordinates Basis Set Obtained by the Essential Dynamics Analysis of Proteins' Molecular Dynamics Simulations. Proteins. 1999; 36:419-424. [PubMed: 10450083]

25. Gerstein M, Altman RB. Average Core Structures and Variability Measures for Protein Families: Application to the Immunoglobulins. J Mol Biol. 1995; 251:161-175. [PubMed: 7643385]

26. Atilgan AR, Durell SR, Jernigan RL, Demirel MC, Keskin O, Bahar I. Anisotropy of Fluctuation Dynamics of Proteins with an Elastic Network Model. Biophys J. 2001; 80:505-515. [PubMed: 11159421]

27. Bahar I, Atilgan AR, Erman B. Direct Evaluation of Thermal Fluctuations in Proteins Using a Single-Parameter Harmonic Potential. Fold Des. 1997; 2:173-181. [PubMed: 9218955] 
28. Dehouck Y, Mikhailov AS. Effective Harmonic Potentials: Insights into the Internal Cooperativity and Sequence-Specificity of Protein Dynamics. PLoS Comput Biol. 2013; 9:e1003209. [PubMed: 24009495]

29. Tama F, Sanejouand YH. Conformational Change of Proteins Arising from Normal Mode Calculations. Protein Eng. 2001; 14:1-6. [PubMed: 11287673]

30. Nishina H, Nimota K, Kukimoto I, Maehama T, Takahashi K, Hoshino S, Kanaho Y, Katada T. Significance of Thr182 in the Nucleotide-Exchange and Gtp-Hydrolysis Reactions of the Alpha Subunit of Gtp-Binding Protein Gi2. J Biochem. 1995; 118:1083-1089. [PubMed: 8749330]

31. Thaker TM, Sarwar M, Preininger AM, Hamm HE, Iverson TM. A Transient Interaction between the Phosphate Binding Loop and Switch I Contributes to the Allosteric Network between Receptor and Nucleotide in Gai1. J Biol Chem. 2014; 289:11331-11341. [PubMed: 24596087]

32. Weinkam P, Chen YC, Pons J, Sali A. Impact of Mutations on the Allosteric Conformational Equilibrium. J Mol Biol. 2013; 425:647-661. [PubMed: 23228330] 
A

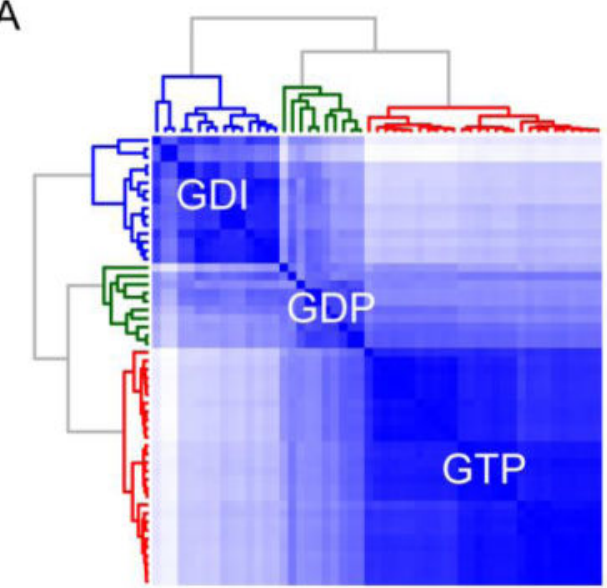

C

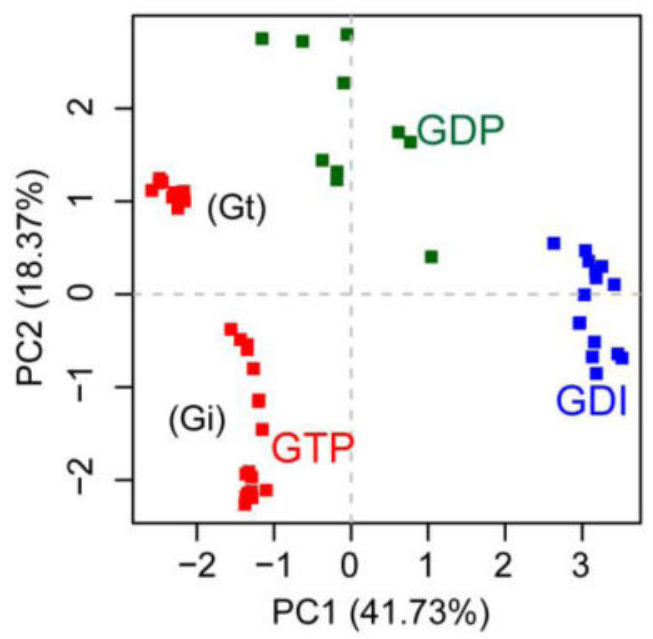

B
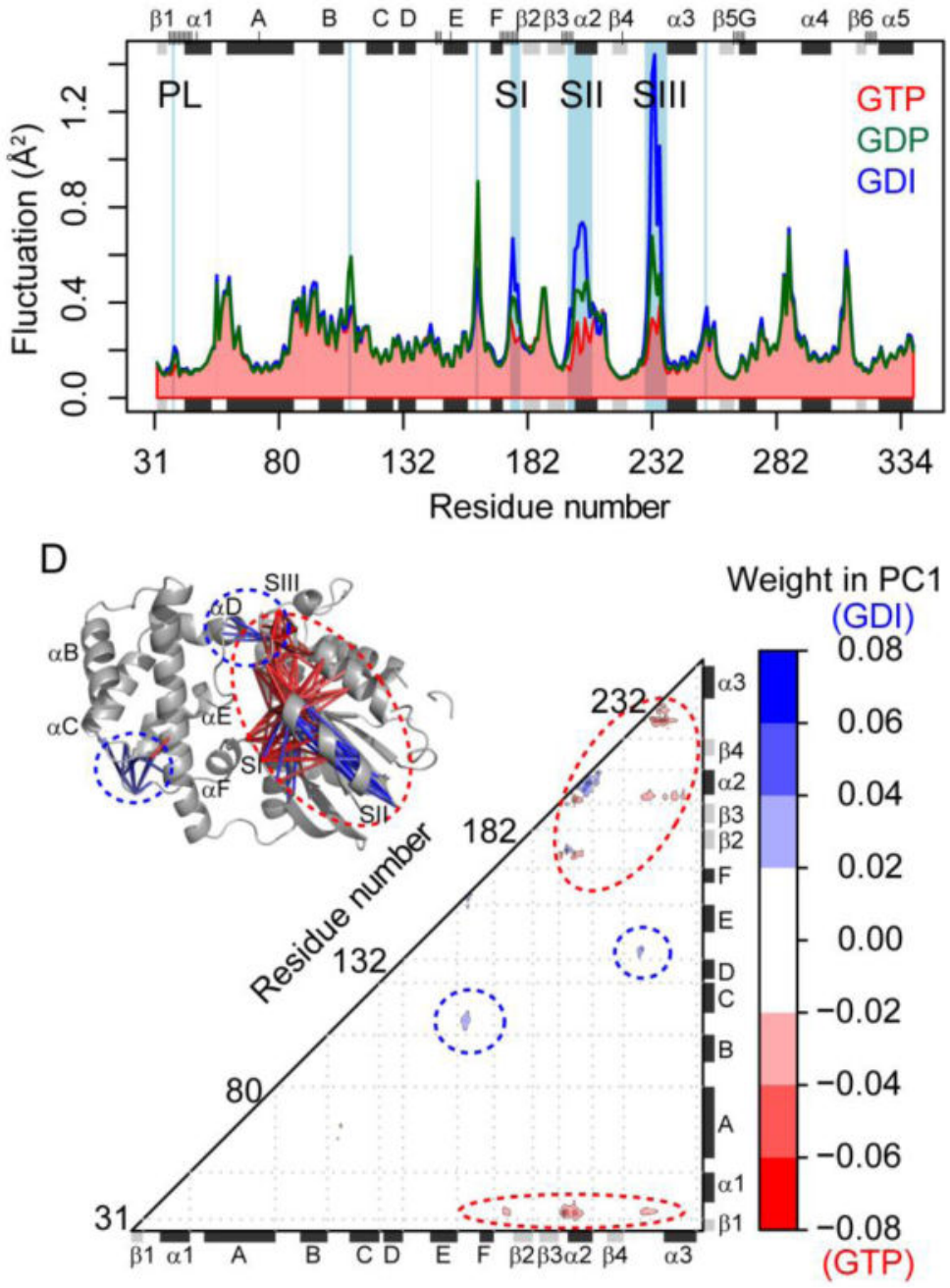

Figure 1. Differences in ensemble NMA predicted local and global dynamics distinguish functional states of $\mathbf{G a}$

(A) Characterization of distinct GTP, GDP, and GDI states from a clustering of NMA derived RMSIP values. (B) Fluctuation analysis reveals structural regions with significantly distinct flexibilities among GTP (red), GDP (green) and GDI (blue) states (sites with a pvalue $<0.05$ are highlighted with a blue shaded background). For each state, ensemble averaged values are shown. Short vertical tick lines at the top of each plot indicate the location of nucleotide binding site residues. (C) Projection of crystallographic structures in the PC1-PC2 subspace of the PCA performed on correlation matrices (See text for details). (D) Identification of residue couplings that characterize specific functional states. Couplings with significantly high (positive) or low (negative) weights in PC1 of the correlation based PCA are indicated with blue and red colors respectively. These couplings are also shown mapped to molecular structure. 

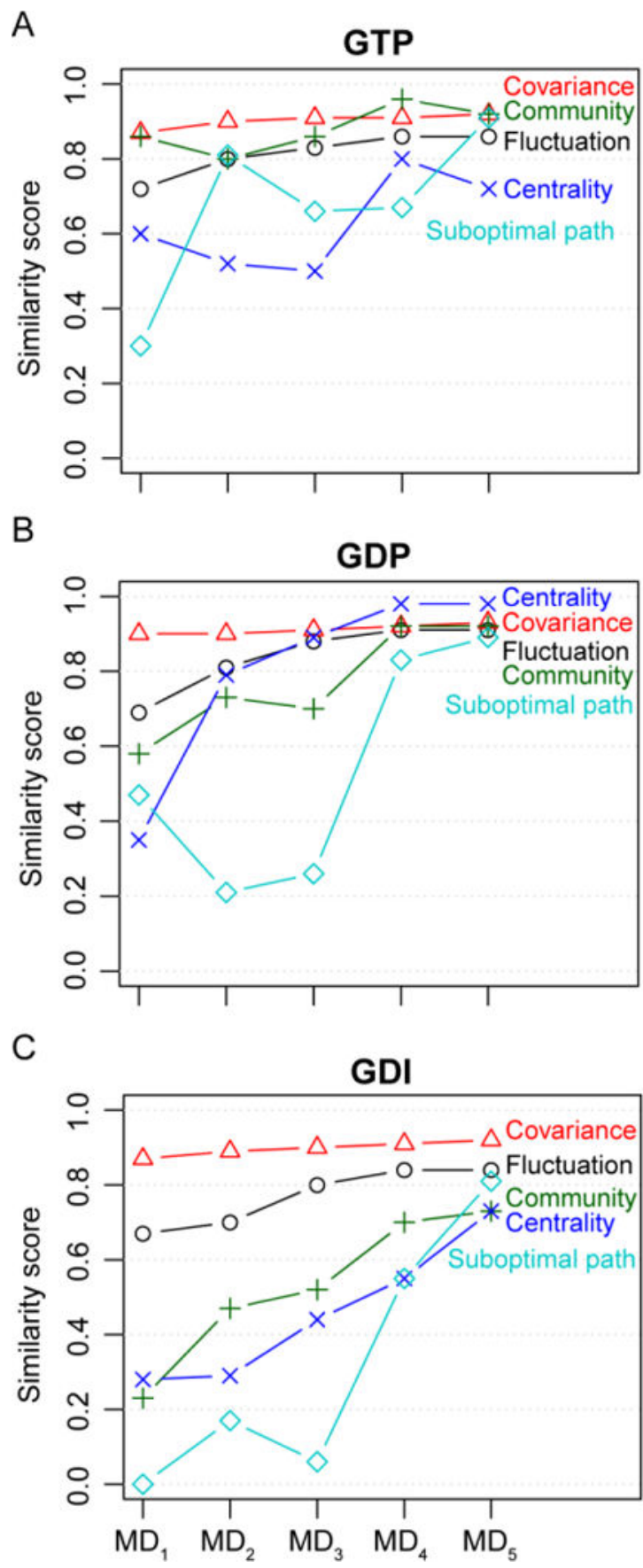

Figure 2. Convergence of structural dynamics in MD simulations

Simulations are performed under three distinct states: GTP (A), GDP (B) and GDI (C). Under each state, five metrics are calculated to compare distinct subsets of simulations: atomic fluctuation, covariance, network community, network node centrality and network suboptimal path. Metric value for $\operatorname{MD}_{n}(n=1,2,3,4$ or 5$)$ is calculated using $n$ independently performed 80-ns simulation trajectories, either simply an ensemble average (fluctuation and covariance) or output of ensemble derived network (community, centrality 
and path; See Methods). Comparison is made between values for the same metric but obtained from two non-overlapping sets of $n$ simulations. 
A

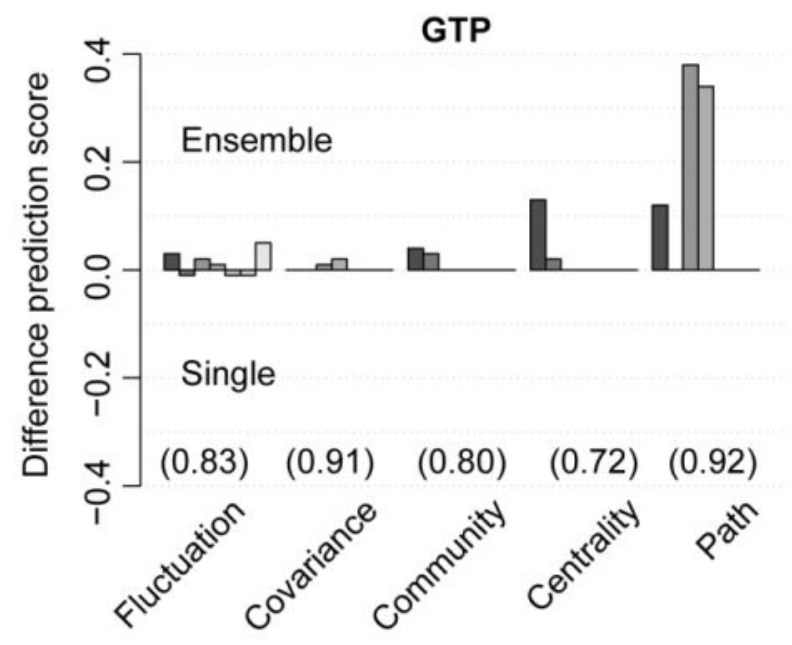

C

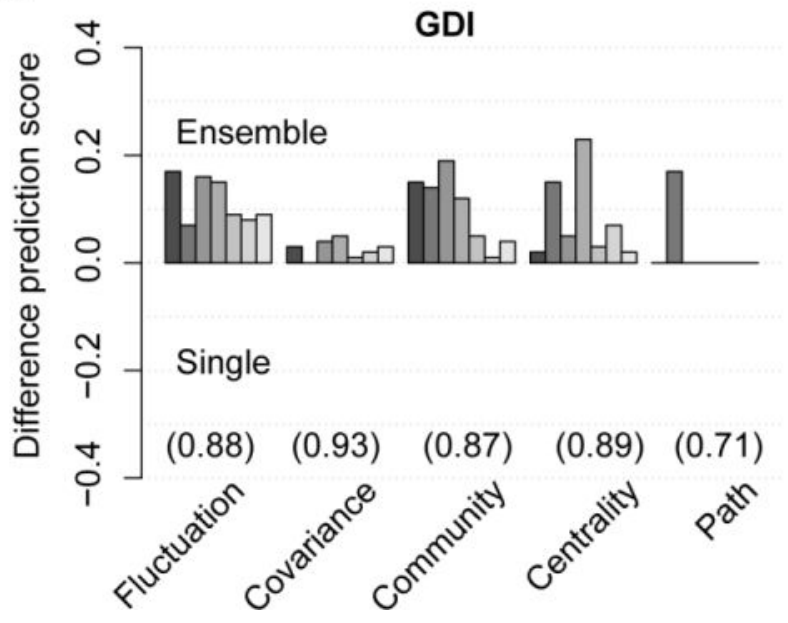

B
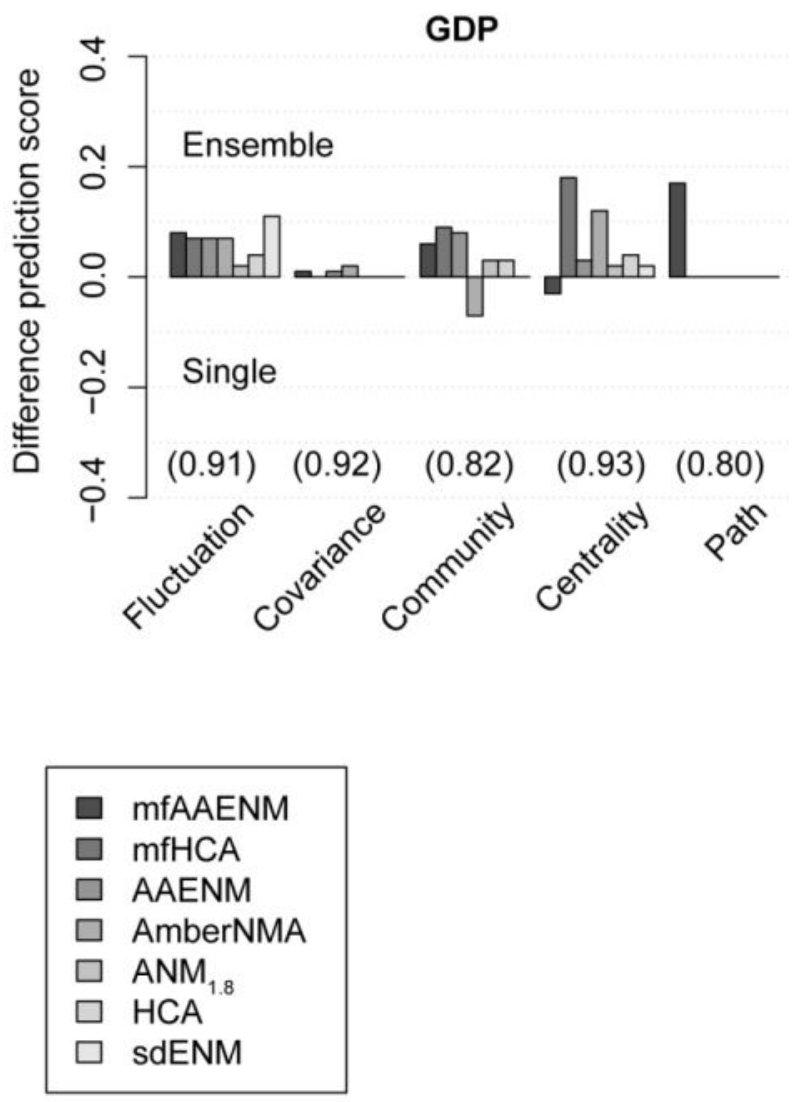

Figure 3. Ensemble NMA has overall improved performance when compared to single structure based NMA

Difference prediction scores of fluctuation, covariance, community, centrality and path between ensemble NMA and single structure NMA are shown for GTP (A), GDP (B) and GDI (C) bound states. Seven methods are considered in the comparison, including six ENMs (mfAAENM, mfHCA, AAENM, ANM ${ }_{1.8}, \mathrm{HCA}$, and sdENM) and the all-atom NMA (AmberNMA). The predominantly positive bars indicate that the ensemble approach has overall improved performance in relation to single structure based calculations.

Comparisons in which both approaches have low prediction scores $(<0.6)$ are excluded. The maximum score across methods for each metric is also shown in parentheses at the bottom of each plot. 

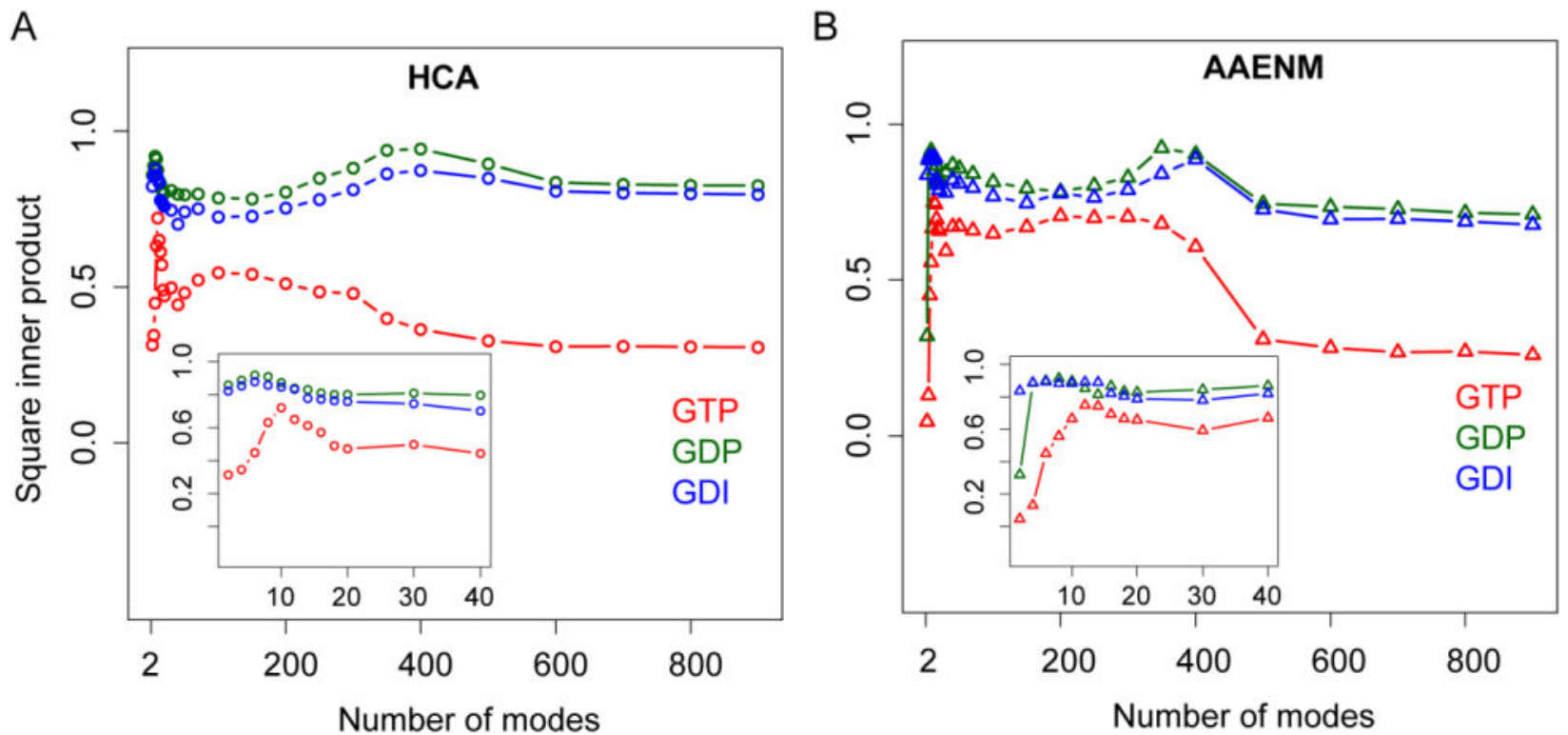

Figure 4. Optimization of the number of modes used for correlation network construction in ENMs

SIP scores are computed between the centralities derived from HCA (A) or AAENM (B) networks and those derived from MD networks. 
A

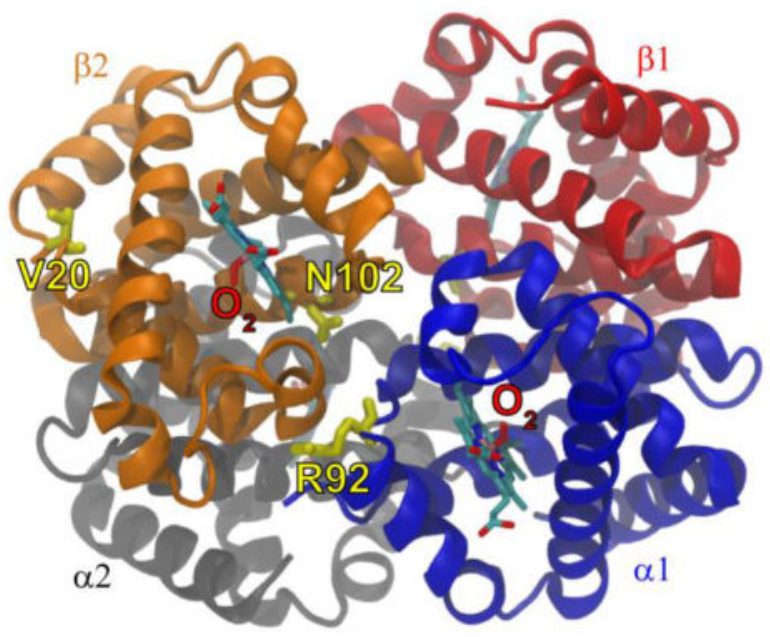

B

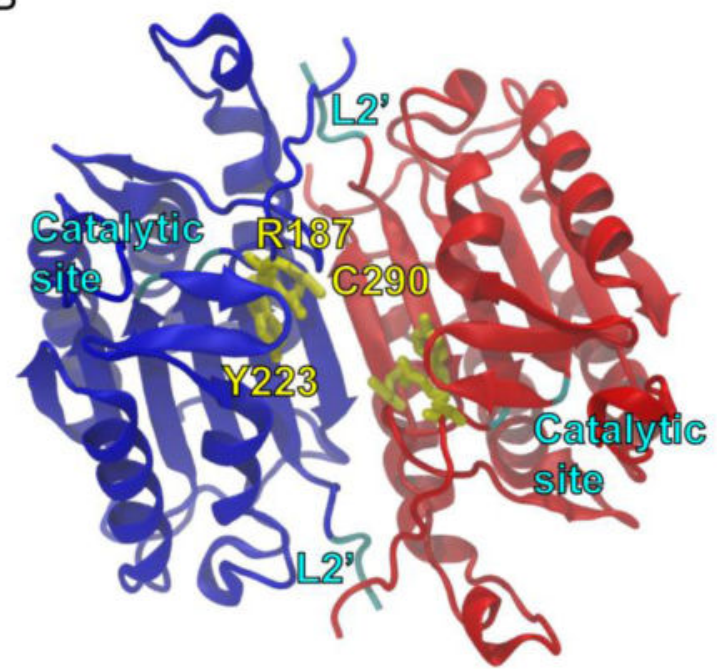

Figure 5. Structures of hemoglobin and caspase 7 with catalytic and allosteric sites shown (A) Hemoglobin tetramer (constructed based on the PDB structure 2DN1) with each subunit displayed in distinct color. The oxygen molecules (red) and experimentally verified allosteric sites (yellow) are also labeled. (B) Caspase 7 homodimer (PDB code 1F1J) with regulatory (C-terminal of L2') and Cys-His dyad catalytic sites colored in cyan. Experimentally verified allosteric sites are depicted as yellow sticks. 


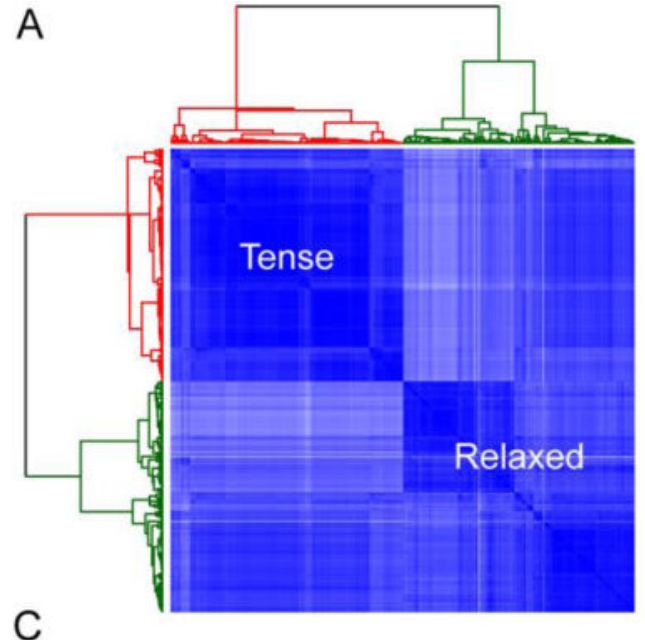

C

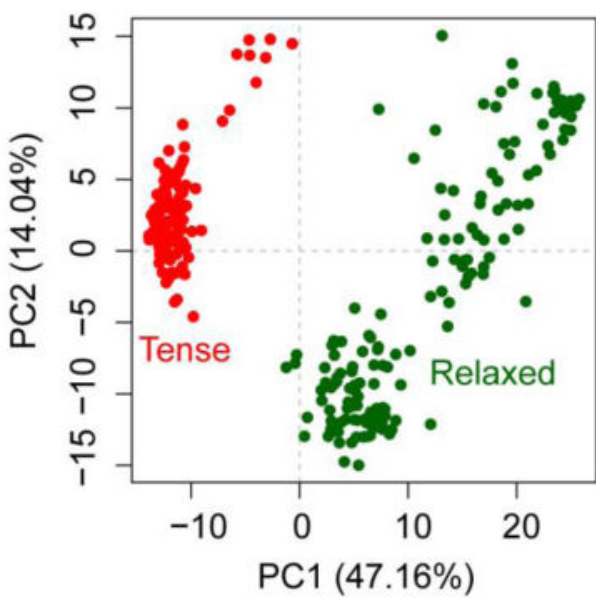

B

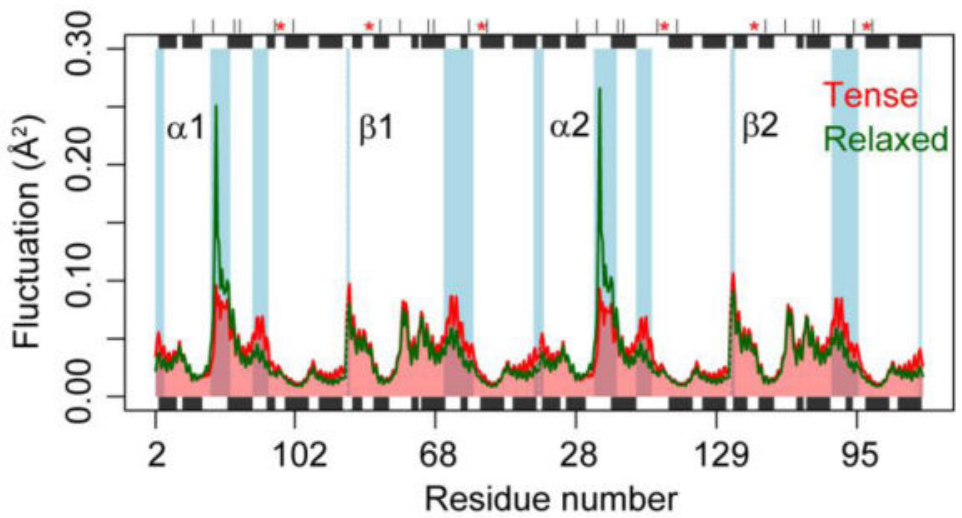

D

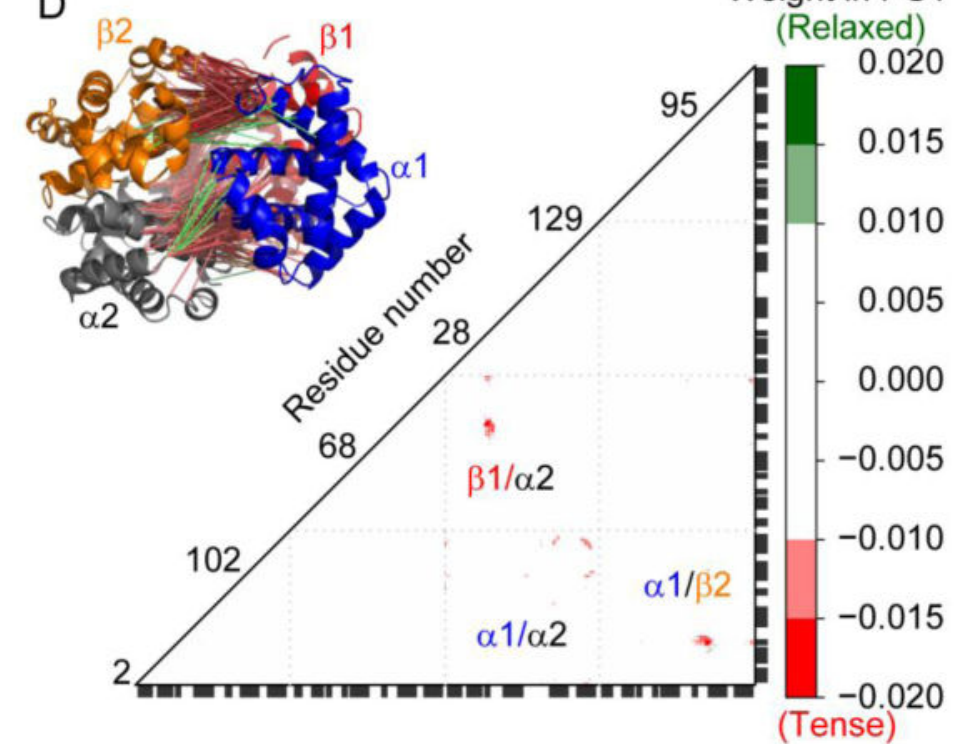

Weight in PC1

(Relaxed)

Figure 6. Ensemble NMA predicts state specific local and global dynamics of hemoglobin (A) Characterization of distinct "Tense" (T) and "Relaxed" (R) states from a clustering of NMA (HCA) derived RMSIP values. (B) Fluctuation analysis reveals structural regions with significantly distinct flexibilities between $\mathrm{T}$ and $\mathrm{R}$ states (sites with a p-value $<0.005$ are highlighted with a blue shaded background). For each state, ensemble averaged values are shown. Short vertical tick lines at the top of each plot indicate the location of heme binding site residues. Red asterisks at the top indicate the experimentally verified allosteric sites. (C) Projection of crystallographic structures in the PC1-PC2 subspace of the PCA performed on correlation matrices. (D) Identification of residue couplings that characterize specific functional states. Couplings with significantly high (positive) or low (negative) weights in PC1 of the correlation based PCA are indicated with green and red colors respectively. These couplings are also shown mapped to molecular structure. 


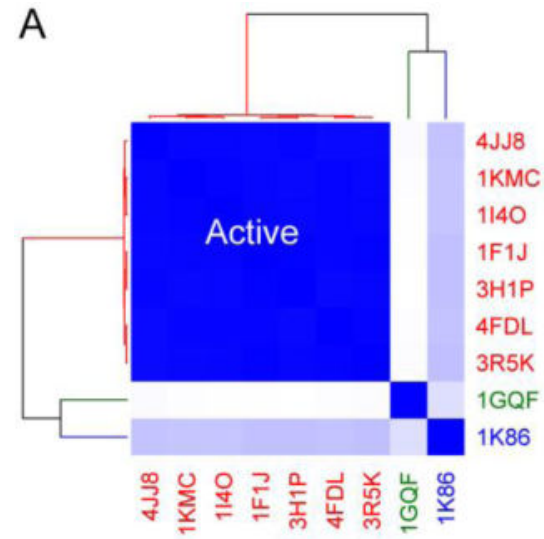

B

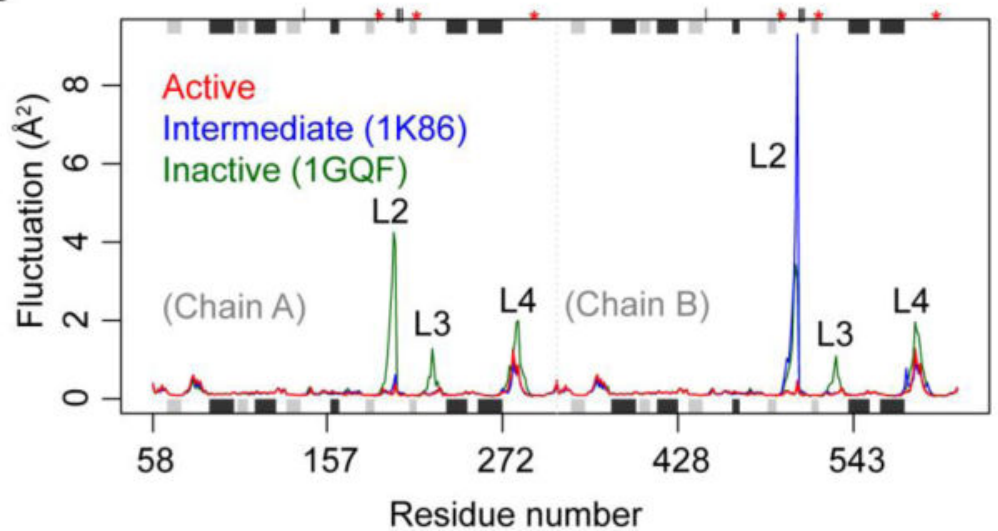

C
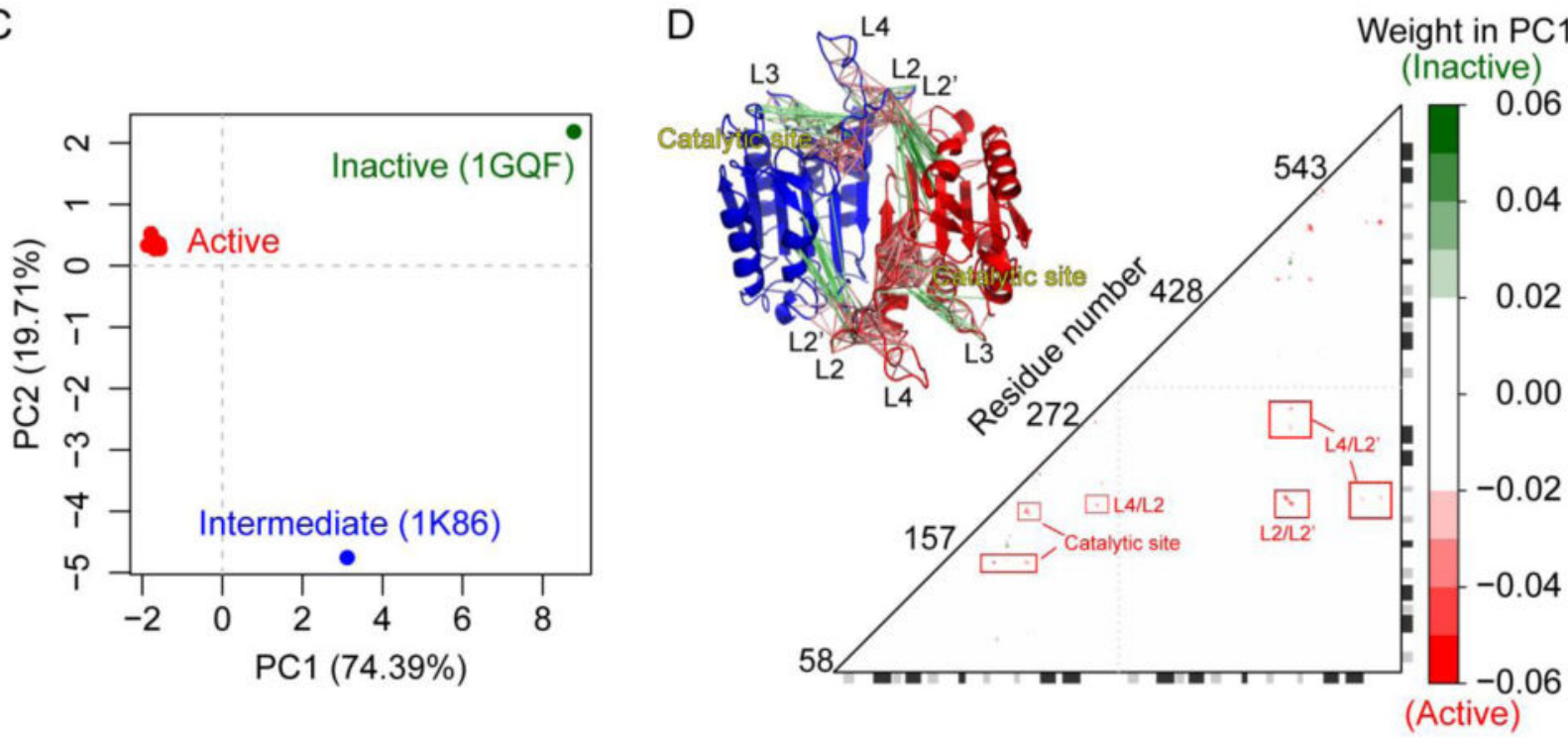

Figure 7. Ensemble NMA predictions for caspase 7

(A) Characterization of distinct "Active", "Intermediate", and "Inactive" states from a clustering of NMA (HCA) derived RMSIP values. (B) Fluctuation analysis reveals structural regions with distinct flexibilities across states. Short vertical tick lines at the top of each plot indicate the location of the loop L2' (regulatory site) and the catalytic site residues. Red asterisks at the top indicate the experimentally verified allosteric sites. (C) Projection of crystallographic structures in the PC1-PC2 subspace of the PCA performed on correlation matrices. (D) Identification of residue couplings that characterize specific functional states. Couplings with significantly high (positive) or low (negative) weights in PC1 of the correlation based PCA are indicated with green and red colors respectively. These couplings are also shown mapped to the molecular structure. 


\section{Table 1}

List of elastic network models tested.

\begin{tabular}{|c|c|c|}
\hline & Feature & Reference \\
\hline $\mathrm{HCA}$ & $\begin{array}{l}\text { Ca based potential; Long range interactions are proportional to an inverse power of six of the } \\
\text { equilibrium distance. }\end{array}$ & Hinsen et al., 2000. \\
\hline varHCA & $\begin{array}{l}\text { The same as HCA except that all force constants are weighted by } w^{10} \text {, where } w \text { is the atomic structural } \\
\text { variance derived from crystallographic structures. }\end{array}$ & Skjaerven et al., 2014. \\
\hline $\mathrm{mfHCA}$ & HCA with fast modes removed (the first 10 slowest modes are kept). & This work. \\
\hline $\mathrm{ANM}_{0.8}$ & Ca based potential; force constants are either 0 or a uniform value within the cutoff distance of $8 \AA$. & Atilgan et al., 2001. \\
\hline $\mathrm{ANM}_{1.8}$ & The same as $\mathrm{ANM}_{0.8}$ except for a larger distance cutoff $18 \AA$. & Atilgan et al., 2001. \\
\hline GNM & $\begin{array}{l}\text { Ca based potential; force constants are either } 0 \text { or a uniform value within the cutoff distance of } 8 \AA \text {; } \\
\text { dynamics is isotropic. }\end{array}$ & Bahar et al., 1997. \\
\hline sdENM & $\begin{array}{l}\text { Ca based potential; force constants are not only dependent on atomic equilibrium distance but amino } \\
\text { acid types. }\end{array}$ & Dehouck et al., 2013. \\
\hline AAENM & $\begin{array}{l}\text { All heavy atoms based potential; force constants are proportional to an inverse power of six of the } \\
\text { equilibrium atomic distance. }\end{array}$ & This work. \\
\hline mfAAENM & AAENM with fast modes removed (the first 400 slowest modes are kept). & This work. \\
\hline
\end{tabular}




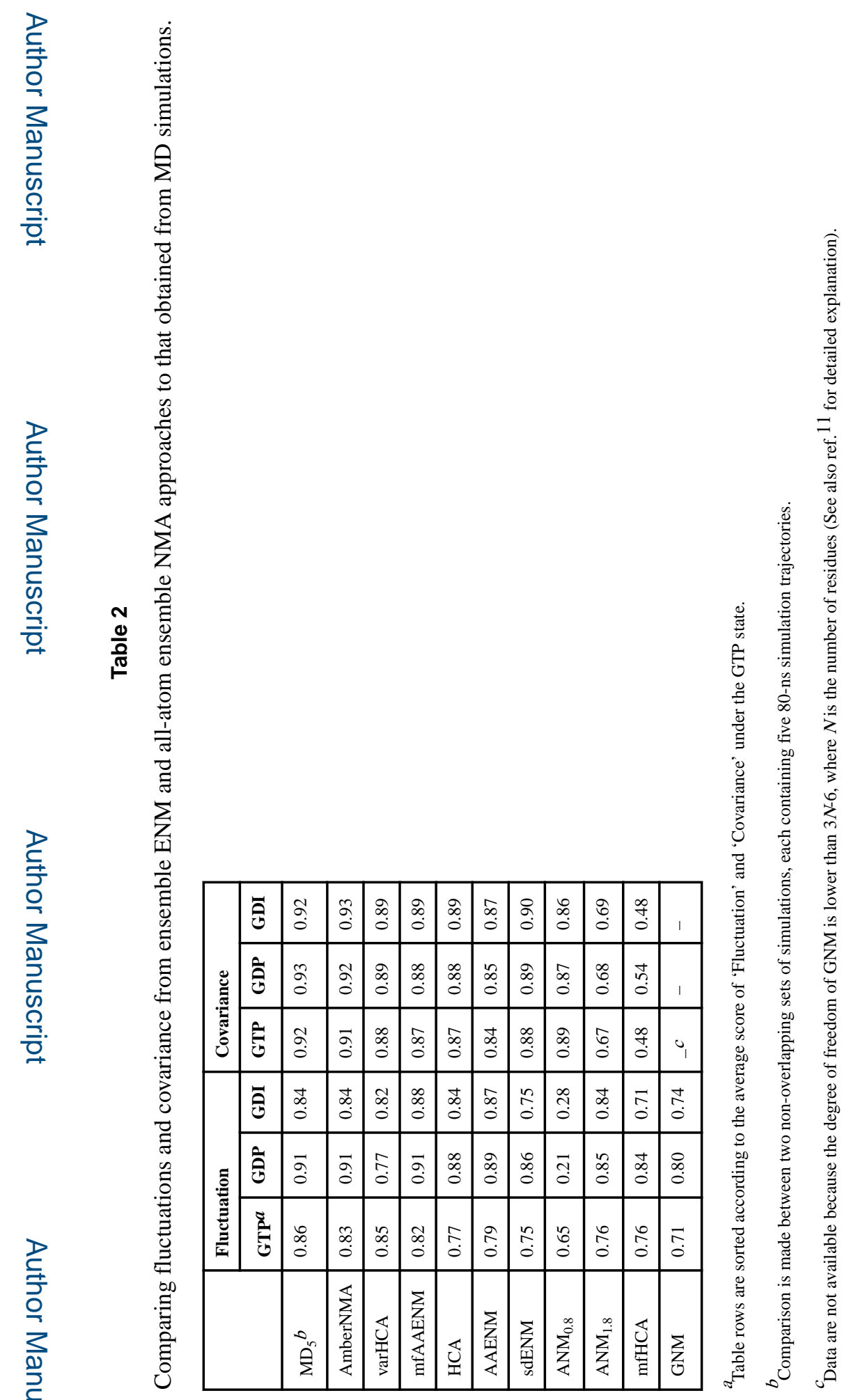



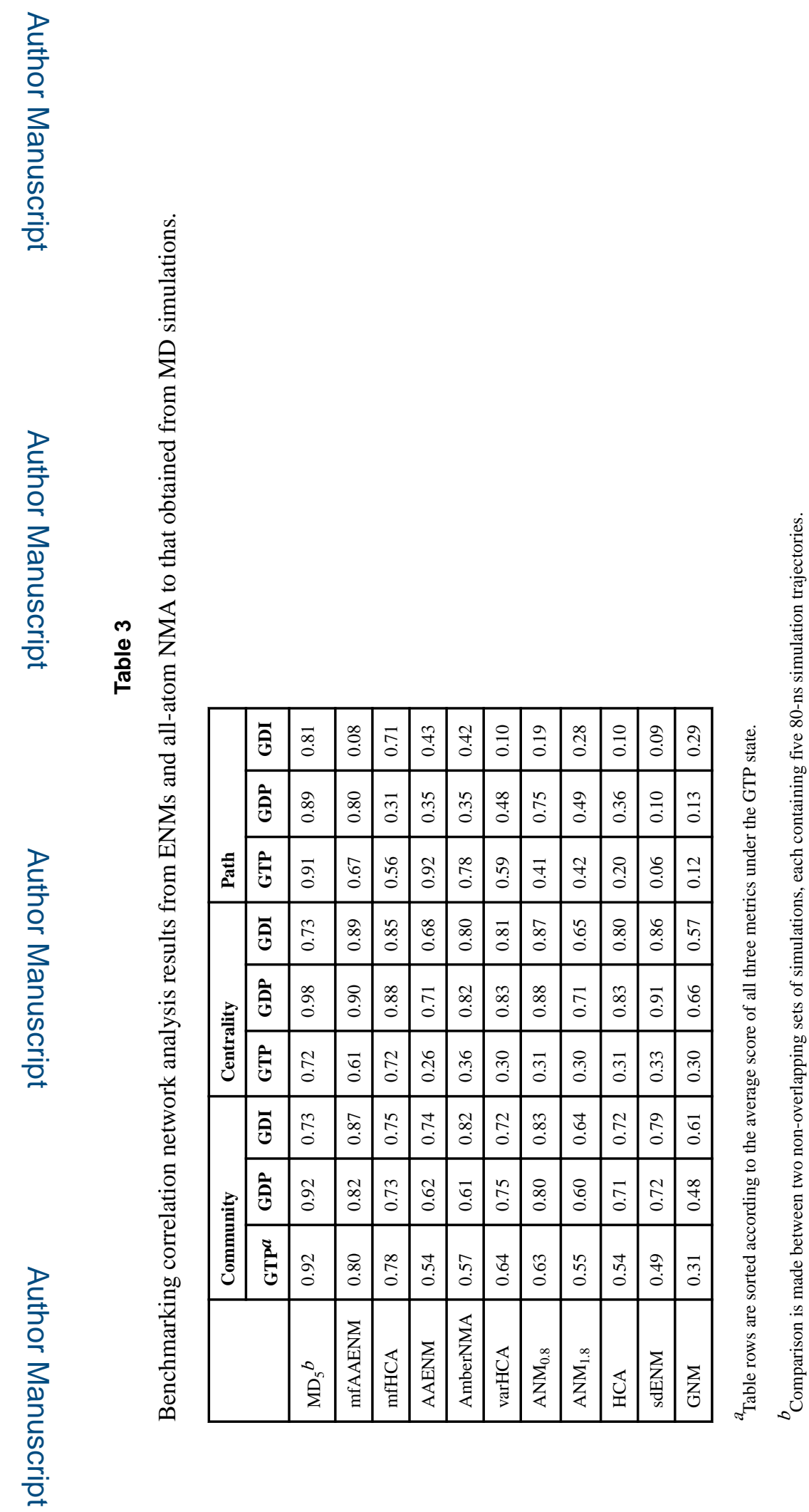
Table 4

Performance of structure and ensemble NMA based network analysis for predicting residues important in hemoglobin and caspase 7 allostery.

\begin{tabular}{|l|l|l|l|l|}
\hline & mfAAENM & AAENM & HCA & Structure network $^{c \mid}$ \\
\hline Hemoglobin (Positive: 6; Negative: 2) $^{a}$ \\
\hline Hit & 4 & 2 & 2 & 0 \\
\hline Significance $^{b}$ & 0.003 & 0.01 & 0.03 & 1.00 \\
\hline False positive & 0 & 0 & 0 & 0 \\
\hline Caspase 7 (Positive: 6; Negative: 0 ) & 2 & 2 & 2 \\
\hline Hit & 4 & 2 & 0.07 & 0.11 \\
\hline Significance & $<0.001$ & 0.06 & \\
\hline
\end{tabular}

${ }^{a}$ Numbers of experimentally confirmed single point mutations that affect (positive) and do not affect (negative) the cooperative oxygen binding in hemoglobin is 6 and 2, respectively. Numbers of experimentally confirmed single point mutations that affect (positive) the allosteric regulation of caspase 7 is 6 . Experimental data are extracted from the ref. ${ }^{32}$. In the literature no negative mutation is reported for caspase 7.

$b_{\text {Si }}$

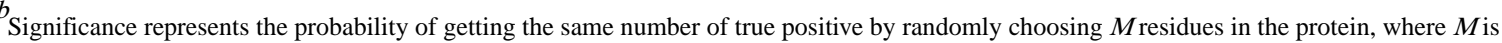
equal to the number of predicted key residues by the model.

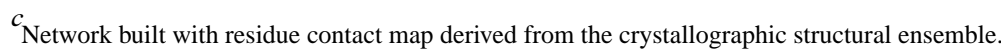

